\title{
A GABAergic Inhibitory Neural Circuit Regulates Visual Reversal Learning in Drosophila
}

\author{
Qingzhong Ren, ${ }^{1,2} \mathrm{Hao} \mathrm{Li,}{ }^{1,2}$ Yanying Wu, ${ }^{1,2}$ Jing Ren, ${ }^{1,2}$ and Aike Guo ${ }^{1,3}$ \\ ${ }^{1}$ Institute of Neuroscience and State Key Laboratory of Neuroscience, Shanghai Institutes for Biological Sciences, Chinese Academy of Sciences, Shanghai \\ 200031, China, ${ }^{2}$ Graduate School of Chinese Academy of Sciences, Beijing 100049, China, and ${ }^{3}$ State Key Laboratory of Brain and Cognitive Science, \\ Institute of Biophysics, Chinese Academy of Sciences, Beijing 100101, China
}

Inflexible cognition and behavior are prominent features of prefrontal cortex damage and several neuropsychiatric disorders. The ability to flexibly adapt cognitive processing and behavior to dynamically changing environmental contingencies has been studied using the reversal learning paradigm in mammals, but the complexity of the brain circuits precludes a detailed analysis of the underlying neural mechanism. Here we study the neural circuitry mechanism supporting flexible behavior in a genetically tractable model organism, Drosophila melanogaster. Combining quantitative behavior analysis and genetic manipulation, we found that inhibition from a single pair of giant GABAergic neurons, the anterior paired lateral (APL) neurons, onto the mushroom bodies (MBs) selectively facilitates behavioral flexibility during visual reversal learning. This effect was mediated by ionotropic $\mathrm{GABA}_{\mathrm{A}}$ receptors in the MB. Moreover, flies with perturbed MB output recapitulated the poor reversal performance of flies with dysfunctional APL neurons. Importantly, we observed that flies with dysfunctional APL-MB circuit performed normally in simple forms of visual learning, including initial learning, extinction, and differential conditioning. Finally, we showed that acute disruption of the APL-MB circuit is sufficient to impair visual reversal learning. Together, these data suggest that the APL-MB circuit plays an essential role in the resolution of conflicting reinforcement contingencies and reveals an inhibitory neural mechanism underlying flexible behavior in Drosophila.

\section{Introduction}

Behavioral flexibility is essential for survival in a changing environment. Reversal learning tasks provide a measure of the hallmark of behavioral flexibility: the ability to shift behavior to altering environmental contingencies (Stalnaker et al., 2009; Brigman et al., 2010; Clarke and Roberts, 2011). In two-option deterministic reversal learning, subjects first learn that one specific stimulus leads to reinforcement (reward or punishment), whereas the alternative stimulus does not. Then, the task contingency is unexpectedly reversed by switching the stimulusreinforcement associations. Although seemingly simple, this task recruits a complex neuronal circuit including orbitofrontal cortex (OFC), striatum, and amygdala in mammals (Stalnaker et al., 2009). Converging evidence indicates that lesions to the OFC result in a specific reversal learning deficit without affecting initial learning in humans (Fellows and Farah, 2003), primates

\footnotetext{
Received Feb. 21, 2012; revised June 11, 2012; accepted July 4, 2012.

Author contributions: Q.R. and A.G. designed research; Q.R., H.L., Y.W., and J.R. performed research; Q.R. and H.L. analyzed data; Q.R. and A.G. wrote the paper.

This work was supported by 973 Program (2011CBA00400 to A.G.), the Natural Science Foundation of China (Grants 30921064, 90820008, and 31130027 to A.G.). We thank Ronald Davis, Zuoren Wang, Bloomington Stock Center, Drosophila Genetic Resource Center, and Vienna Drosophila RNAi Center for generously providing fly stocks and reagents and Martin Heisenberg and MuMing Poo for critical reading of the manuscript and helpful comments. We also thank the two anonymous reviewers for thoughtful comments, which greatly improved our manuscript.

The authors declare no competing financial interests.

Correspondence should be addressed to Dr. Aike Guo, Institute of Neuroscience, State Key Lab of Neuroscience, Shanghai Institutes for Biological Sciences, Chinese Academy of Sciences, 320 Yue Yang Road, Shanghai 200031, China.E-mail:akguo@ion.ac.cn.

DOI:10.1523/JNEUROSCI.0827-12.2012

Copyright $\odot 2012$ the authors $\quad 0270-6474 / 12 / 3211524-15 \$ 15.00 / 0$
}

(Iversen and Mishkin, 1970; Dias et al., 1996), and rodents (Schoenbaum et al., 2003). In addition, deficit in reversal learning is a prominent feature of several debilitating neuropsychiatric disorders that are characterized by OFC abnormalities (Chamberlain et al., 2008; Stalnaker et al., 2009; Brigman et al., 2010). However, neither the precise role of the OFC nor the circuit mechanism of reversal learning is fully understood in mammals (Schoenbaum et al., 2009).

Insects can also perform reversal learning tasks (Menzel, 1969; Quinn et al., 1974; Tully et al., 1990; Komischke et al., 2002). In honeybees, one study found that blockade of the output of the mushroom bodies (MBs) impaired olfactory reversal learning without affecting initial learning (Devaud et al., 2007). This result indicates that these two learning processes may recruit partially distinct neuronal machineries even in insects and that the MBs may contribute to information processing during reversal learning. In Drosophila, the MBs have been considered as a predominant locus for simple forms of olfactory learning (Schwaerzel et al., 2002; Heisenberg, 2003; McGuire et al., 2005; Keene and Waddell, 2007). Interestingly, one recent study found that signaling activity of Rac, a small G protein involved in cytoskeleton dynamics, in a subset of Drosophila MB neurons is critical for olfactory reversal learning but not for initial learning (Shuai et al., 2010). Despite this progress, however, the neural mechanism underlying reversal learning in Drosophila remains poorly understood, especially at the circuitry level.

In the present study, we first established a robust visual reversal learning paradigm to study behavioral flexibility and demonstrated that flies could flexibly switch their preference toward 
visual patterns when stimulus-reinforcement associations reverse. Previous research has shown that visual learning and memory in the flight simulator relies acutely on cAMP signaling in the central complex neuropil (Liu et al., 2006). The MBs, while dispensable for simple visual learning (Wolf et al., 1998), are implicated in a variety of visual cognitive behaviors (Heisenberg, 2003). Therefore, we assayed whether the MB neuropil is necessary for visual reversal learning. Our results identified the anterior paired lateral (APL) neurons-MB circuit as a brain center selectively promoting visual reversal learning.

\section{Materials and Methods}

Fly strains. Flies were reared in standard food vials at $25^{\circ} \mathrm{C}$ and $60 \%$ relative humidity with a $12 \mathrm{~h}$ light/dark cycle (Guo et al., 1996). The upstream activating sequence (UAS) strain Rdli8-10j (Liu et al., 2007) was provided by Dr. R. Davis (The Scripps Research Institute, Jupiter, FL) and the GH146-Gal4 (Stocker et al., 1997) and UAS-TNTE (Sweeney et al., 1995) strains by Z.R. Wang (Institute of Neuroscience, Chinese Academy of Sciences). The NP2631 and NP3061-Gal4 strains (Tanaka et al., 2008) were obtained from the Drosophila Genetic Resource Center, the Tub-Gal80 ${ }^{\text {ts }}$ strains (\#7017 and 7019) (McGuire et al., 2003) from the Bloomington Stock Center, and the UAS-GBRi (v1784) and UAS$G A D_{\text {RNAi }}$ (v32344) strains (Dietzl et al., 2007) from Vienna Drosophila RNAi Center. Other strains used in this study were extant lines in the lab.

Visual learning paradigm. Briefly, female flies (3-7 d old) were immobilized by cold anesthesia. Then, they were glued (Locktite UV glass glue) by their heads and thoraxes to triangular-shaped hooks. Individual flies were then kept in small chambers overnight and fed with sucrose solution. After a $12-20 \mathrm{~h}$ recovery period, they were separately mounted onto the flight simulator (Reichardt and Wenking, 1969; Heisenberg and Wolf, 1979; Wolf and Heisenberg, 1991). Flies were tethered in the center of a circular panorama with four visual patterns distributed evenly in the center of the four quadrants. The T-shaped patterns measure $40^{\circ}$ both vertically and horizontally; the bars of the Ts measure $12^{\circ}$ wide. The patterns with different contour orientations were left or right tilted bars $\left(40^{\circ}\right.$ long and $12^{\circ}$ wide). Most flies did not exhibit a pronounced bias toward one of these two patterns, while a small proportion showed moderate preference. In very rare cases, if a fly showed very strong biases toward one pattern, the fly was discarded before training began. The yaw torque signals generated by the flies were detected by a torque meter (Götz, 1964), which then drive the rotation of the panorama via a negative feedback mechanism. This arrangement should allow flies to stabilize and choose their flight orientations with respect to the visual patterns by adjusting their yaw torque. A computer continuously recorded (sampling frequency $20 \mathrm{~Hz}$ ) and stored the flight orientation (panorama position) for further analysis. During the training sessions, heat punishment was provided by an infrared laser beam $(\lambda=10600 \mathrm{~nm})$, aiming at the fly's abdomen from above and behind. The power of the laser is $42 \mathrm{~mW}$, measured by a laser power meter (Model LP-102A, Chinese National Institute of Metrology). The heat was switched on whenever the flight orientation fell inside the punished quadrants and was switched off in the safe quadrants. To eliminate the effects of spontaneous pattern preference, we used a reciprocal training design. For instance, when the T-shaped patterns were used, half of the flies were trained to avoid upright $\mathrm{T}$ and the other half to avoid inverted T. A fly's performances were quantified over variable time bins using the performance index (PI), which is defined as $\left(t_{1}-t_{2}\right) /\left(t_{1}+t_{2}\right)$, where $t_{1}$ is the time flies spent in the safe quadrants, and $t_{2}$ is the time flies spent in the punished quadrants. Thus, a PI $=1$ means that flies spent the entire time period within the safe quadrant, while a PI $=0$ indicates that flies spent equal time in the safe and punished quadrants. PIs were averaged across individual flies for further statistical analysis.

Behavioral assay. The standard "learning" task consisted of several sessions: pretraining test (6 min), training session (10 min, including 8 min training and a 2 min interim test), and short-term memory test (4 $\min )$. In the "reversal learning" task, a second reversal training period was included before the final memory test. In learning under "low punishment," the training intensity was set to $66.7 \%$ or $33.3 \%$ of previous experiments by reducing the power of the infrared laser, while the duration of the heating remained unchanged. For the "extinction" task, the final test session of standard learning were prolonged to $14 \mathrm{~min}$. For the "differential conditioning" task in Figure $7 \mathrm{~A}$, we first trained flies using patterns with different contour orientation (left or right tilted bars), and then using patterns with different vertical positions of center of gravity (upright or inverted Ts).

Immunohistochemistry. Adult female flies $\left(3-7 \mathrm{~d}\right.$ after eclosion at $\left.25^{\circ} \mathrm{C}\right)$ were collected, and brains were dissected in ice-cold PBS $(1.86 \mathrm{~mm}$ $\mathrm{NaH}_{2} \mathrm{PO}_{4}, 8.41 \mathrm{~mm} \mathrm{Na}_{2} \mathrm{HPO}_{4}$, and $175 \mathrm{~mm} \mathrm{NaCl}$ ) and fixed in $4 \%$ paraformaldehyde solution in PBS for $1 \mathrm{~h}$ at room temperature. After three 20 min washings in PBS containing $0.3 \%$ Triton X-100 (PBT), the brains were blocked with 5\% normal goat serum (Invitrogen 01-6201) in PBT for an hour. Brains were then incubated with primary antibodies in blocking solution at $4^{\circ} \mathrm{C}$ for $48 \mathrm{~h}$. After six 20 min washings in PBT, samples are incubated with secondary antibodies at $4^{\circ} \mathrm{C}$ for $12 \mathrm{~h}$. The primary antibodies used include: rabbit anti-RDL (1:100; kindly provided by Dr. R. Davis, The Scripps Institute, Jupiter, FL) (Liu et al., 2007), mouse anti-FasII (1:50; Developmental Studies Hybridoma Bank 1D4), rabbit anti-GABA (1:50; Sigma A2052), mouse anti-green fluorescent protein (GFP) (1:100; Invitrogen A11120), mouse anti-nc82 (1:40; Developmental Studies Hybridoma Bank ), and rabbit anti-GFP (1:100; Sigma A6455). Secondary antibodies (1:500; Invitrogen) include: Alexa 488 goat anti-rabbit IgG (A11008), Alexa 488 goat anti-mouse IgG (A11001), Alexa 568 goat anti-rabbit IgG (A11011), Alexa 633 goat antimouse IgG (A21053), and Alexa 633 goat anti-rabbit IgG (A21070). Finally, brains were washed with PBT six times and mounted in Vectashield (Vector Laboratories).

Confocal microscopy and quantification of immunofluorescence. Wholemount fly brains were scanned with a Nikon FN1 confocal microscope and image stacks were taken at $1 \mu \mathrm{m}$ intervals using a $20 \times$ Plan Apochromat objective lens $(\mathrm{NA}=0.75)$ at $1024 \times 1024$ pixel resolution. To quantify the downregulation of GABA in the APL neurons, or the downregulation of $\mathrm{RDL}$ in the MBs, images belonging to the same comparison group were obtained under the same settings (gain, offset, laser power, and pinhole size) on the confocal microscope. The grayscale intensities of confocal images were analyzed over an intensity scale from 0 to 4095 (minimum to maximum intensity for 12-bit images, respectively) using the ImageJ software (National Institutes of Health). The images shown in the quantification experiments were average projections of 15-30 sections.

$M B$ ablation. We followed the procedure described previously (de Belle and Heisenberg, 1994). In brief, wild-type flies (3-7 d old) lay their eggs on agar plates containing sugar and grape juice for an hour. The eggs were placed at $25^{\circ} \mathrm{C}$ for $16-18 \mathrm{~h}$, and newly hatched larvae (within 30 $\mathrm{min}$ ) were quickly transferred into a yeast suspension containing $50 \mathrm{mg}$ $\mathrm{ml}^{-1}$ hydroxyurea to ablate the MB neuroblasts. The yeast suspension for the vehicle group did not contain hydroxyurea. After4 $\mathrm{h}$ of treatment, larvae were transferred into fresh food vials.

Temporal control of gene expression. We used the temporal and regional gene expression targeting (TARGET) system (McGuire et al., 2003) to control transgene expression. The experimental group was raised the permissive temperature (PT; in a cubicle where the temperature is held constant at $\left.18^{\circ} \mathrm{C}\right)$. Within $24 \mathrm{~h}$ after eclosion from the pupae, these flies were transferred to the restrictive temperature (RT; in an incubator where the temperature is held above $30^{\circ} \mathrm{C}$ ). The control group was kept at the PT throughout development and adulthood. Under PT or RT, flies were cultured in $60 \%$ relative humidity and a $12 \mathrm{~h}$ light/dark cycle. Incubation at RT lasted $72 \mathrm{~h}$ for induction of UAS- $G A D_{\mathrm{RNAi}}$ expression, and $12 \mathrm{~h}$ for induction of UAS-TNTE expression. Both the experimental and control groups were left at $25^{\circ} \mathrm{C}$ for adaptation just before the behavioral experiments.

Quantitative PCR. We extracted RNA from 30-50 flies with TRIzol (Invitrogen) and assessed the quality of RNA using the Lab-on-a-Chip 2100 Bioanalyzer (Agilent) platform. Then, $2 \mu \mathrm{g}$ of total RNA was treated with RQ1 DNase (Promega) and reverse transcribed using oligo(dT) primers and Superscript III reverse transcriptases (Invitrogen). Realtime PCR was performed with SYBR Premix Ex TaqTM II kit (Takara) using an ABI PRISM 7000 real-time PCR Detection system (Applied Biosystems). We calculated the relative mRNA level using the compara- 
tive $\mathrm{C}_{\mathrm{T}}$ method (Pfaffl, 2001). Three repeats were run for each sample and the values are normalized to an rp49 as a control gene.

Primer sequence used were as follows: qPCR rp49: $5^{\prime}$-CCAAGGACTTCA TCCGCCACC-3', 5' -GCGGGTGCGCTTGTTCGATCC-3' and CG6706: 5'-CGACATGTTTCTATACCGGCTC-3', 5' -CTTTCGATCCGATAGC ACCAG-3'.

Statistical analyses. Data were shown as means \pm SEM. We used onesample $t$ test (two-sided $p$ value) to compare the PIs against the chance level (Liu et al., 1999; Brembs and Wiener, 2006). Two-sample $t$ tests were used for comparison between two groups. Comparison between multiple groups was done by one-way ANOVA followed by planned pairwise comparisons with Tukey-HSD post hoc test. The effect of genotype on extinction performance was analyzed by repeated-measures ANOVA, with block number as the within-subjects factor. About $10 \%$ of the PIs in this study do not approximate normal distributions (ShapiroWilks test). For statistical analysis of data that include such PIs, we used nonparametric tests including Wilcoxon test, Mann-Whitney test, and Kruskal-Wallis test followed by Dunn's multiple comparisons. Statistical analysis was performed using Origin 8 (Origin Lab). The sample size of each group is reported in the figure legends and the significance levels of post hoc tests are shown in the figures. Asterisks indicate levels of significant differences $\left({ }^{*} p<0.05 ;{ }^{* *} p<0.01 ;{ }^{* * *} p<0.001\right)$.

\section{Results}

\section{Visual reversal learning in Drosophila}

To explore behavioral flexibility in Drosophila, we first established a robust visual reversal learning paradigm. An individual fly was presented with two sets of visual patterns distributed evenly in the four quadrants of the visual panorama in the flight simulator (Fig. 1A; see Materials and Methods). Flight orientation toward one set of patterns (e.g., inverted T) was coupled with undesirable heat punishment while the other set (upright $\mathrm{T}$ ) was not. The flies gradually developed a robust avoidance of the pattern associated with punishment during the initial training session (Fig. $1 B$, blocks 4, 5, 7, and 8). The stimulus-reinforcement contingency was reversed immediately after the initial training by reversing the pattern associated with heat punishment. To adjust their behavior appropriately, flies should now fly toward the previously punished pattern, instead of avoiding it. We observed that, as shown in Figure $1 B$, the preference of the flies indeed switched after contingency reversal. We quantified flies' choices using PI over 2 min bins, calculated by the time flies spent in the safe quadrants minus that in the punished quadrants, and divided by the total time (Fig. $1 B$ ). The mean PIs of the reversal training session (blocks 9, 10, 12, and 13) and the final test session (blocks 14 and 15 ) were $0.46 \pm 0.05$ and $0.22 \pm 0.08$, respectively, indicating that flies spent significantly more time in the safe quadrants (Wilcoxon test, $Z=5.09, p<0.001$, and one-sample $t$ test, $t_{(41)}=2.81, p=0.007$, respectively) (Fig. $1 C$ ).

Importantly, the above results depended on neither the specific fly strain nor the specific visual feature used in the test: the same results were obtained for the Canton-S (CS) fly strain and for visual patterns that differed by contour orientation (left or right tilted bar) (Fig. 1C). For CS flies, the reversal training and test PI were $0.47 \pm 0.06$ and $0.31 \pm 0.07$ (one-sample $t$ test, $t_{(31)}=$ 4.72, $p<0.001$ and $t_{(31)}=8.40, p<0.001$, respectively). For contour orientation patterns, the reversal training and test PI were $0.34 \pm 0.05$ and $0.19 \pm 0.07$ (Wilcoxon test, $Z=4.72, p<$ 0.001 and one-sample $t$ test, $t_{(47)}=2.57, p=0.013$, respectively). Together, these data indicate that flies showed flexibility in response to changes in stimulus-reinforcement contingency.

\section{The APL neurons are required for visual reversal learning}

We next considered the neuronal substrates implementing behavioral flexibility during visual reversal learning in Drosophila.
In each brain hemisphere, $\sim 2500$ Kenyon cells $(\mathrm{KC})$ form a prominent structure called the MBs, which can be further divided into the calyx, where $\mathrm{KC}$ dendrites receive input, and the vertical ( $\alpha$ and $\alpha^{\prime}$ ) and horizontal $\left(\beta, \beta^{\prime}\right.$, and $\gamma$ ) lobes, where $\mathrm{KC}$ axons terminate (Strausfeld et al., 1998; Tanaka et al., 2008). They have been critically implicated in olfactory learning and memory (Davis, 2005; Keene and Waddell, 2007; Heisenberg and Gerber, 2008), along with a variety of other behaviors, including context generalization (Liu et al., 1999), visual attention (van Swinderen and Greenspan, 2003), habit formation (Brembs, 2009), and decision making (Zhang et al., 2007). In honeybees, blocking $\alpha$-lobes of the MBs by local injection of procaine impairs acquisition of olfactory reversal learning, without affecting initial learning (Devaud et al., 2007). Therefore, we focused our attention on the MB neuropil. Interestingly, the MBs in Drosophila are innervated by a single pair of giant GABAergic neurons, the APL neurons (Tanaka et al., 2008; Liu and Davis, 2009). Clonal analysis of the GH146-Gal4 revealed that the projection of the APL neuron formed a continuous net-like innervation throughout the compartment of the ipsilateral MB except the cell body region (Liu and Davis, 2009). They inhibit olfactory learning (Liu and Davis, 2009), but promote stabilization of labile olfactory memory traces (Pitman et al., 2011; Wu et al., 2011).

To determine whether the APL neurons contribute to visual reversal learning, we reduced GABA synthesis in these two neurons by expressing siRNA targeting glutamic acid decarboxylase (GAD), which catalyzes GABA synthesis, using the GH146-Gal4 driver line. As visualized by membrane-localized mCD8-GFP expression (Fig. 2A), the GH146-Gal4 labels the APL neurons (Liu and Davis, 2009), cholinergic projection neurons (PNs) of the antennal lobe (Stocker et al., 1997), and certain neurons with projection to the optic lobe (Heimbeck et al., 2001). This $G A D_{\mathrm{RNAi}}$ strategy, when compared with other neuronal silencing methods such as tetanus toxin (TNT)-mediated blockade (Sweeney et al., 1995) or temperature-sensitive Shibire expression (Kitamoto, 2001), should permit relatively specific disruption of the inhibitory function of the APL neurons. To verify the efficacy of this strategy, we performed immunohistochemistry experiments to quantify the amount of GABA in the APL neurons in flies carrying the GH146-Gal4 driver alone, and in flies carrying both the GH146-Gal4 and Gad-RNAi transgenes (Fig. 2B). Consistent with a previous report (Liu and Davis, 2009), we found that GABA immunoreactivity was significantly reduced in the cell body region of the APL neuron in GH146-Gal4; Gad-RNAi flies, compared with that in control flies (two-sample $t$ test, $t_{(30)}=$ 4.36, $p<0.001$ ) (Fig. 2C).

We examined the performance of GH146-Gal4/+; Gad$\mathrm{RNAi} /+$ group and genetic control groups in visual learning (Fig. $2 D$ ) and reversal learning (Fig. $3 A$ ) assay. All groups performed normally in short-term memory test, measured immediately after standard training (Fig. $2 E$ ), and the initial learning phase of the reversal task (Fig. 3B1, left) (both $p>0.05$ ). These results indicate that Gad-RNAi expression did not significantly affect basic visual processing or motor control that was required for our behavioral paradigm. The performance of GH146-Gal4/+; Gad$\mathrm{RNAi} /+$ flies and genetic control flies in the reversal learning phase are shown in Figure 3, $A 1$ and $A 2$. While the control groups exhibited reversal in their preference in response to the new stimulus-punishment association, the GH146-Gal4/+; GadRNAi/ + flies exhibited little preference toward the current safe pattern and showed markedly lower PIs during both reversal training and test (ANOVA, $F_{(2,49)}=6.08, p=0.004$ and $F_{(2,49)}=$ $8.54, p<0.001$, respectively) (Fig. 3B2, B3, left). 
A

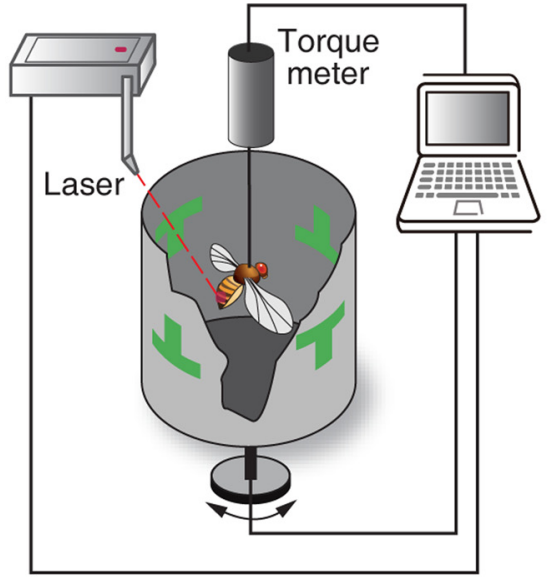

B
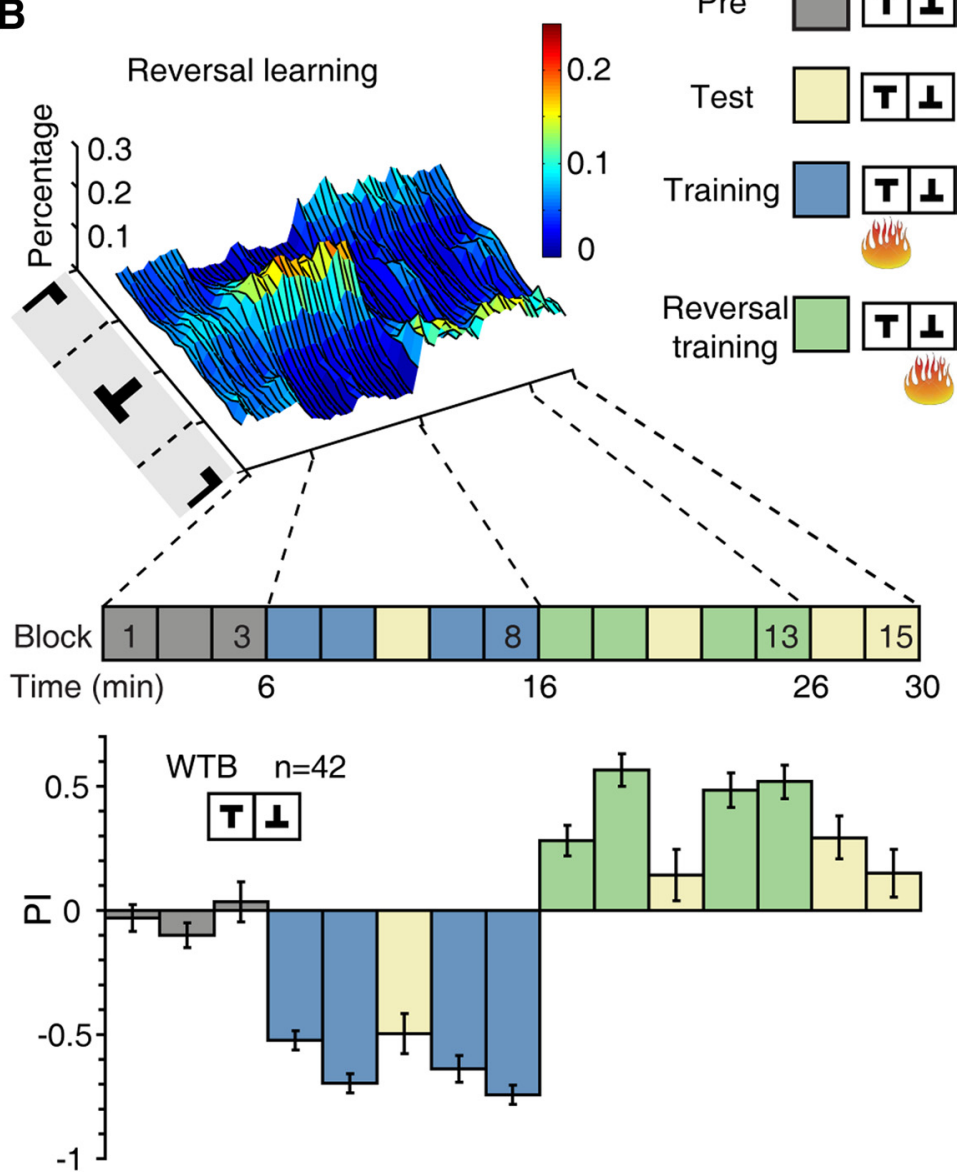

C

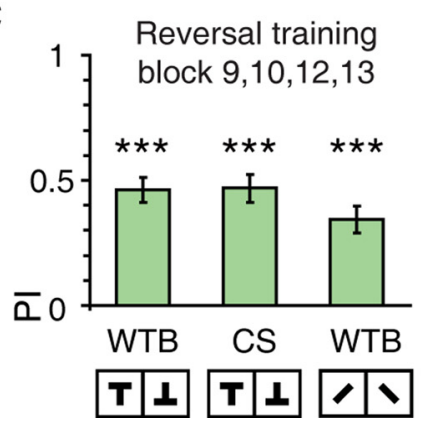

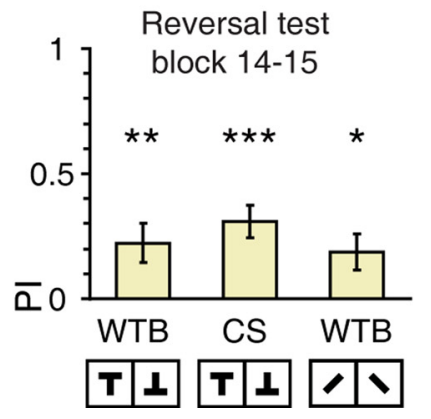

Figure 1. Visual reversal learning in Drosophila. $A$, The visual flight simulator. Tethered flies can control their flight orientations relative to the visual patterns by generating yaw torque, which was measured by the torque meter and controlled the rotation of the panorama. During training, flies are heated by a laser beam when orienting toward one of the patterns. $\boldsymbol{B}$, Top, surface plot of the percentage of time wild-type Berlin (WTB) flies spent orienting toward different flight directions in the "reversal" protocol.
To further rule out the contribution of PNs, we tested another Gal4 line, NP2631-Gal4 (Fig. 2F), which labels the APL neurons as well as other neurons in the protocerebrum, but not PNs (Tanaka et al., 2008). Quantification of GABA staining revealed that GABA immunoreactivity was significantly decreased in the APL cell body when the Gad-RNAi transgene was combined with the NP2631-Gal4 (twosample $t$ test, $t_{(22)}=5.18, p<0.001$ ) (Fig. $2 G, H)$. Similar to that of GH146-Gal4, decreased GABA synthesis in NP2631-Gal4expressing neurons also lead to a severe deficit, specifically in reversal training and test (Mann-Whitney test, $U=28, p<$ 0.001 and $U=51, p<0.001$, respectively) (Fig. 3B2,B3, right). We did not observe any significant effect on short-term memory (Fig. 2I) or initial learning (Fig. 3B1, right) (Mann-Whitney test, both $p>$ $0.05)$.

The arborization patterns of GH146Gal4 and NP2631-Gal4 in the optic lobe were different (Fig. $2 J, K$ ). The medulla and the lobula plate were heavily innervated in NP2631-Gal4 (Fig. 2J), whereas such innervations were absent in GH146Gal4 (Fig. $2 \mathrm{~K}$ ). Although both lines showed expression in the lobula, the arborization pattern of NP2631-Gal4 was mostly restricted to the inner layers of the lobula (Fig. 2J), while the arborization pattern of GH146-Gal4 was sparse and distributed, without obvious layer localization (Fig. $2 \mathrm{~K}$ ). These observations do not support Gad-RNAi expression in the visual system as the main cause of the reversal learning defect.

To further test our hypothesis of the involvement of the APL neurons in reversal learning, we sought to determine whether GABA receptors that mediate the inhibitory effects of the APL neurons are required for reversal learning. Previous reports have shown that downregulation of GABA synthesis in the APL neurons led to enhanced olfactory associative learning in the T-maze paradigm (Liu and Davis, 2009), the same behavioral phenotype produced by knocking down $\mathrm{GABA}_{\mathrm{A}}$ receptor RDL in the MBs (Liu et al., 2007).

\section{$\leftarrow$}

Heat punishment was associated with upright $T$ but switched to inverted T upon reversal of stimulus-reinforcement association. Bottom, Pls of WTB flies in the "reversal" protocol. $C$, Wild-type flies (WTB or CS) showed significantly positive PIs during reversal training and test. Patterns with different vertical position of center of gravity or contour orientation were used. $n=32-48$ flies per group. Data were shown as means \pm SEM. ${ }^{*} p<0.05 ;{ }^{* *} p<0.01 ;{ }^{* * *} p<0.001 . n$, the total number of flies examined. 


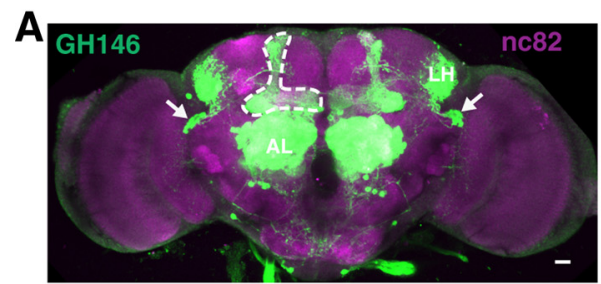

D
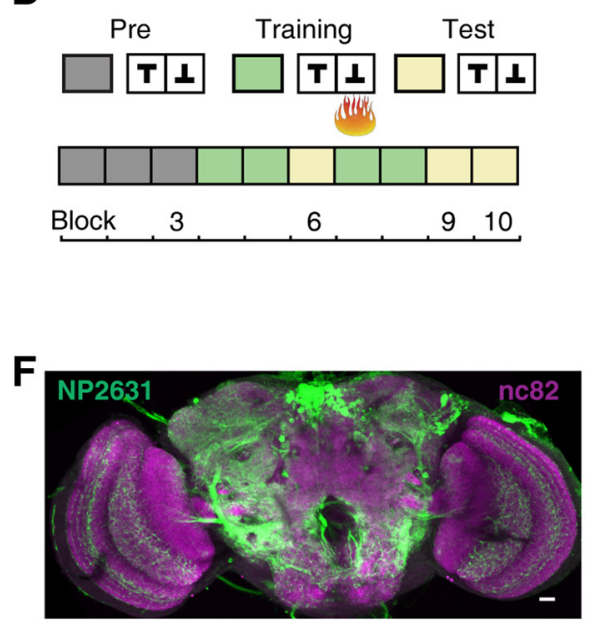

G
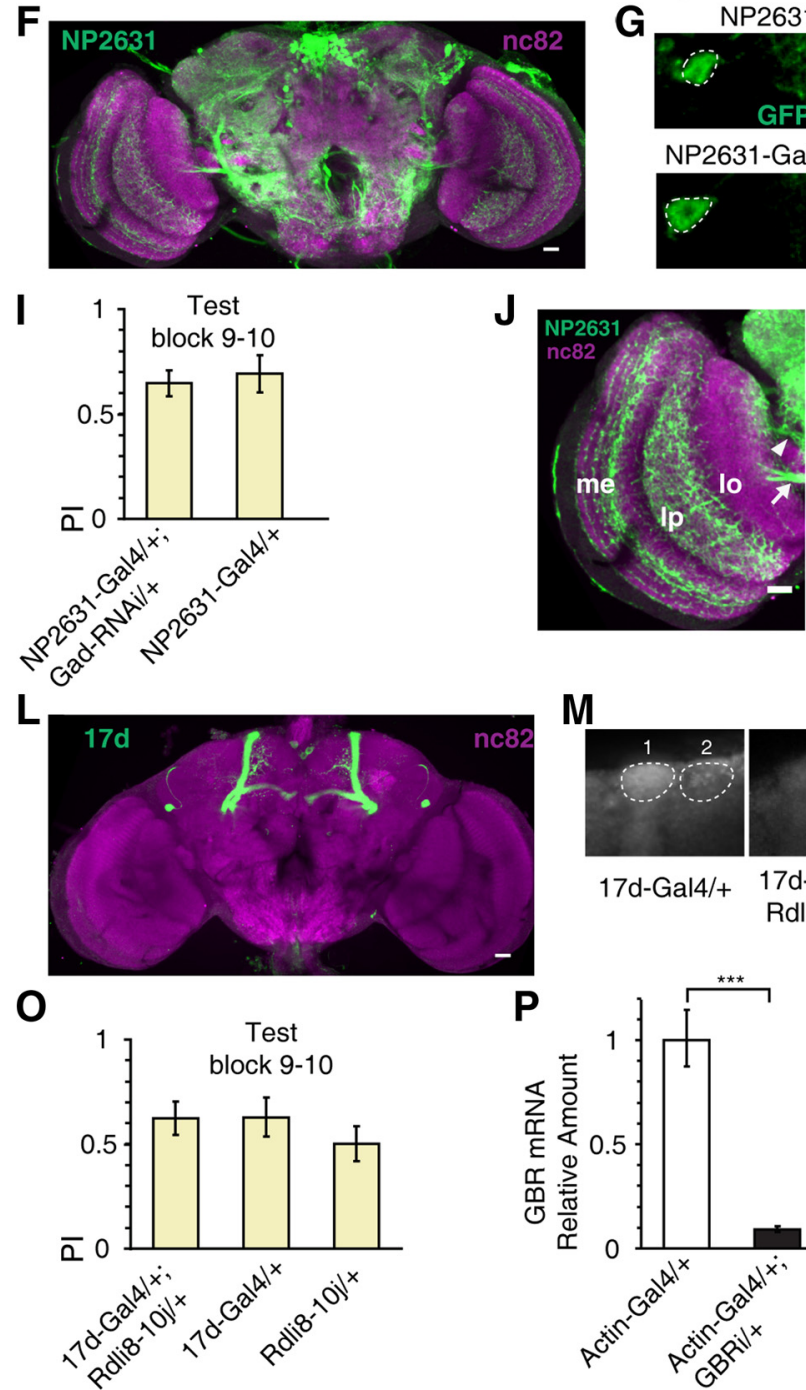

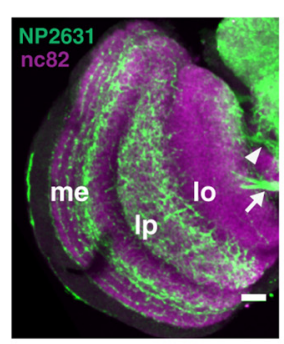

M

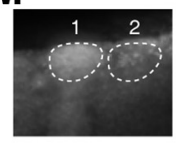

17d-Gal4/+
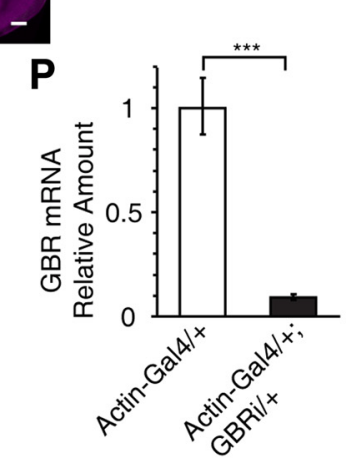

B

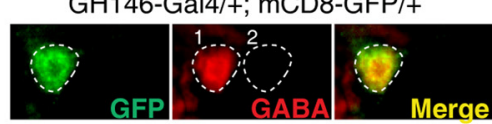

GH146-Gal4/+; mCD8-GFP/Gad-RNAi

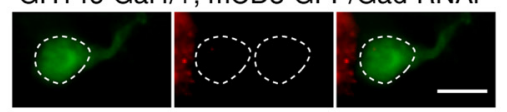

E

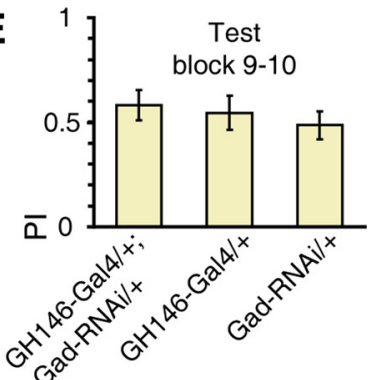

C

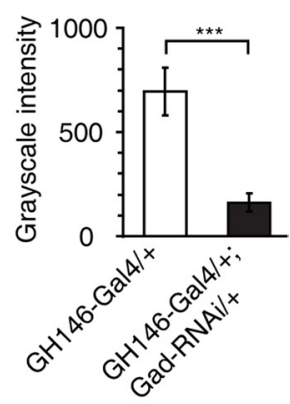

H

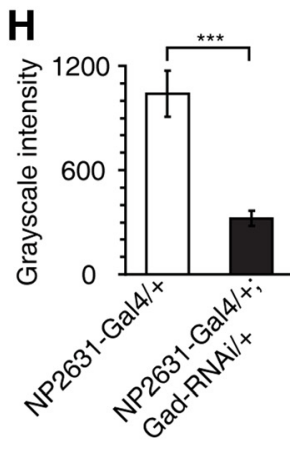

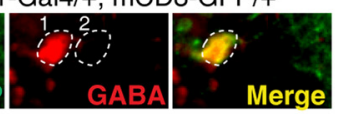

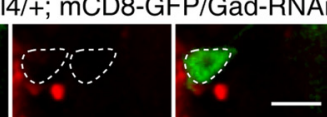

K

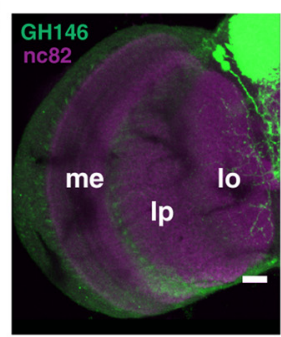

N

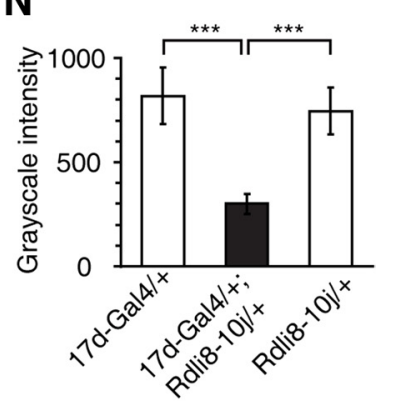

Figure 2. The APL neurons are dispensable for visual short-term memory. A, Adult expression pattern of the GH146-Gal4 enhancer trap line visualized by Gal4-dependent mCD8-GFP expression (green). Neuropils were counterstained with anti-nc82 antibody (magenta). Arrows indicate the cell bodies of the APL neurons. The ramification pattern of the APL neuron in the MB is outlined in dashed line. In addition to APL, the GH146 also labels projection neurons in the antennal lobe (AL). These neurons project to MB calyx and lateral horn (LH). $B$, Representative confocal images showing the immunoreactivity of GABA and GFP in the APL cell body in GH146-Gal4/+; mCD8-GFP/+ flies, or in GH146-Gal4/+; mCD8-GFP/Gad-RNAiflies. Left, Antibody staining for GFP. Middle, Antibody staining for GABA. Right, A merged view of the left and middle image. Colocalization of GFP with GABA is shown in yellow. The level of GABA immunoreactivity was calculated as the grayscale intensity $(0-4095)$ of the APL cell body (dashed circle 1) after subtracting that of a background region (dashed circle 2). $C$, Quantification of the downregulation of GABA synthesis in APL cell body via Gad-RNAi expression in GH146-Gal4. $n=16$ samples per genotype. D, Training protocol for measuring visual short-term memory. $\boldsymbol{E}$, Reducing GABA synthesis in GH146-Gal4expressing neurons had no effect on visual short-term memory. $n=16-18$ flies per group. $\boldsymbol{F}$, Projection of confocal stacks showing the adult expression pattern of the (Figure legend continues.) 
Given that the APL neurons ramify only within the ipsilateral MB and use the inhibitory neurotransmitter GABA (Tanaka et al., 2008; Liu and Davis, 2009), we expected that GABA receptor signaling in $\mathrm{MB}$ neurons may also be indispensable for normal visual reversal learning performance.

In Drosophila MBs, the $\mathrm{GABA}_{\mathrm{A}}$ receptor subunit RDL is expressed throughout the substructures except the cell body area (Liu et al., 2007), whereas the $\mathrm{GABA}_{\mathrm{B}}$ receptor $\mathrm{R} 2$ $\left(\mathrm{GABA}_{\mathrm{B}}-\mathrm{R} 2\right)$ subunit is expressed only in calyx (Enell et al., 2007). Of the various MB Gal4 lines, we focused our attention principally on the $17 \mathrm{~d}-$ Gal4 (Fig. $2 \mathrm{~L}$ ), which drives expression in the $\mathrm{MB} \alpha / \beta$ lobe, especially the $\alpha / \beta_{\mathrm{c}} \mathrm{KC}$ subtype (Aso et al., $2009)$. To probe the function of GABA receptors, we downregulated the expression of $R D L$ and $G_{A B A}-R 2$ via RNAi [using UAS-Rdli8-10j (Liu et al., 2007) and UAS-GBRi (Dietzl et al., 2007) strains, respectively]. The downregulation of the RDL receptor was confirmed by immunohistochemical experiments (Fig. $2 M$ ). When the UAS-Rdli8-10j transgene was combined with 17d-Gal4, the RDL immunoreactivity exhibited a significant reduction in the tip of MB $\alpha$ lobe (KruskalWallis test, $H=14.9, \mathrm{df}=2, p<0.001$ ) (Fig. $2 N$ ). We also checked the effectiveness of the UAS-GBRi strain at the mRNA level. Quantitative PCR showed that the relative amount of GBR mRNA was significantly reduced in Actin-Gal4/+, GBRi/+ flies comparing to the Actin-Gal4/+ flies (twosample $t$ test, $\left.t_{(24)}=9.94, p<0.001\right)$ (Fig. $2 P$ ). In the behavioral experiments, we found that neither manipulation of the GABA receptors had any effect on short-term memory (Fig. $2 O, Q$ ) or initial learning (Fig. 3C1) (all $p>0.05$ ). However, RDL knockdown severely impaired performance during reversal training and test (Kruskal-Wallis test, $H=16.5$, $\mathrm{df}=2$, $p<0.001$ and ANOVA, $F_{(2,27)}=7.84, p=0.001$, respectively) (Fig. 3C2,C3, left). By comparison, $\mathrm{GABA}_{\mathrm{B}}-\mathrm{R} 2$ knockdown had no such effect ( $t$ test or Mann-Whitney test, both $p>$ 0.05 ) (Fig. 3C2,C3, right).

Together, the above results were most consistent with the notion that reducing GABA synthesis in the APL neurons disrupted behavioral flexibility during visual reversal learning and this ef-

\section{$\leftarrow$}

(Figure legend continued.) NP2631-Gal4 (green). Neuropils were counterstained with antinc82 antibody (magenta). The NP2631-Gal4 does not label projection neurons in the antennal lobe. $\mathbf{G}$, Same as in $\boldsymbol{B}$, except that the genotypes were NP2631-Gal4; mCD8-GFP/+ and NP2631-Gal4; mCD8-GFP/Gad-RNAi. $\boldsymbol{H}$, Quantification of the downregulation of GABA synthesis in APL cell body via Gad-RNAi expression in NP2631-Gal4. $n=16$ samples per genotype. $I$, Reducing GABA synthesis in NP2631-Gal4-expressing neurons had no effect on visual short-term memory. $n=16$ flies per group. $\boldsymbol{J}, \boldsymbol{K}$, The expression patterns of the NP2631 and GH146-Gal4 in the optic lobe. The medulla (me) and lobula plate (Ip) were densely labeled in NP2631-Gal4, but not in GH146-Gal4. In NP2631-Gal4, one bundle of neurites (arrowhead) entered the lobula at the neck and mainly innervated the inner layers of lobula. $J$, Arrow indicates neurite bundles projecting to the lobula plate. The arborization of the GH146Gal 4 in the lobula is sparse and distributed. L, Adult expression pattern of the $17 \mathrm{~d}-\mathrm{Ga} / 4$ (green). Neuropils were counterstained with anti-nc82 antibody (magenta). In addition to the $M B \alpha / \beta$ lobe, the $17 \mathrm{~d}-\mathrm{Gal} 4$ also labels a pair of large neurons (not APL), which mainly project to the superior and inferior protocerebrum. $\boldsymbol{M}$, Representative confocal images of anti-RDL immunofluorescence in 17d-Gal4/+ ; UAS-Rdli8 -10j/ + flies and genetic control flies. The level of RDL expression was calculated as the grayscale intensity of $\alpha$ lobe tip (dashed circle 1) minus that of a nearby background region (dashed circle 2). $n=12$ samples per genotype. $N$, Quantification of the downregulation of RDL in MB $\alpha$ lobe tip. $\boldsymbol{0}$, Downregulation of RDL in MB $\alpha / \beta$ lobe had no effect on visual short-term memory. $n=14-18$ flies per group. $\boldsymbol{P}$, The effectiveness of the $G A B A_{B}-R 2$ subunit RNAi line. The results were normalized to levels of the ribosomal protein Rp49. $Q$, Downregulation of $G A B A_{B}$ receptor $R 2$ subunit in $M B \alpha / \beta$ lobe had no effect on visual short-term memory. $n=10-12$ flies per group. GBRi: GABA $A_{B}$ receptor R2 subunit RNAi. Data were shown as means \pm SEM. ${ }^{*} p<0.05 ;{ }^{* *} p<0.01 ;{ }^{* *} p<0.001$. Scale bars, $20 \mu \mathrm{m}$. fect might be mediated by fast-acting ionotropic $\mathrm{GABA}_{\mathrm{A}}$ receptors in the $\mathrm{MB} \alpha / \beta$ lobe.

\section{The MBs are involved in visual reversal learning}

Since the projections of the APL neurons are intrinsic to the MBs (Tanaka et al., 2008), we further investigated whether synaptic output of $\mathrm{MB}$ neurons is necessary for reversal learning. We blocked synaptic transmission from the $\mathrm{MB} \alpha / \beta$ lobe via expression of the light chain of TNT (TeTxLC; UAS-TNTE) (Sweeney et al., 1995), which cleaves synaptobrevin (a synaptic vesicle membrane protein) in $17 \mathrm{~d}-$ Gal4. Consistent with a previous report (Liu et al., 1999), we found that 17d-Gal4/TNTE flies showed normal short-term memory (Fig. $4 A$, left) and initial learning (Fig. $4 B$, left) (ANOVA, both $p>0.05$ ). In contrast, performance during reversal training and test was significantly impaired in these 17d-Gal4/TNTE flies as compared with genetic control flies (Kruskal-Wallis test, $H=12.8, \mathrm{df}=2, p=0.002$ and ANOVA, $F_{(2,61)}=9.49, p<0.001$, respectively) (Fig. $4 C, D$, left).

To confirm the involvement of the MB neurons, we further combined the UAS-TNTE transgene with the NP3061-Gal4 (Fig. $4 E)$. This Gal4 line drives expression all over the $\alpha / \beta$ lobes of the MBs, while the expression outside the MBs is minimal among the 25 MB Gal4 lines examined in a previous study (Aso et al., 2009). We found that blocking synaptic transmission from MB neurons defined by the NP3061-Gal4 had little detectable effects on shortterm memory (Fig. $4 A$, middle) or initial learning (Fig. $4 \mathrm{~B}$, middle) (ANOVA or Kruskal-Wallis test, both $p>0.05$ ). However, this manipulation led to a severe deficit during reversal training and test (ANOVA, $F_{(2,43)}=6.86, p=0.003$ and $F_{(2,43)}=9.90, p<$ 0.001 , respectively) (Fig. $4 C, D$, middle). Thus, the output of $\mathrm{MB}$ neurons to APL or other neurons is necessary for reversal learning. Chemical ablation of the MBs (using hydroxyurea; Fig. $4 F, G)$ (de Belle and Heisenberg, 1994) also led to a selective deficit in reversal training and test (two-sample $t$ test, $t_{(46)}=$ $-2.95, p=0.005$ and $t_{(46)}=-3.83, p<0.001$, respectively) (Fig. $4 C, D$, right), whereas having little impact on short-term memory (Fig. $4 A$, right) or initial learning (Fig. $4 B$, right) (two-sample $t$ test, both $p>0.05)$. Together, our results imply that the APL neurons and the MBs form an inhibitory circuit that regulates behavioral flexibility in Drosophila.

\section{A high-resolution analysis of flight behavior}

We first examined the flight paths of individual flies in the visual operant conditioning task. We observed that in the pretest session, wild-type flies frequently changed their flight directions around the four quadrants (Fig. 5A). In the training period, the flight behavior was characterized by the avoidance of punished quadrants and long dwelling time in the safe quadrants (Fig. 5A). Post-training test session showed that flies continued to exhibit a strong preference toward the safe pattern (Fig. $5 A$ ). Note that flies sometimes "accidentally" entered and quickly turned away from the punished quadrant, rather than simply persisted in their heading from training to test (Fig. 5A). Careful examination of individual traces of flies with dysfunctional APL-MB circuit did not reveal gross abnormalities in flight behavior (Fig. $5 B$; data not shown).

In the process of reversal learning, wild-type flies avoided the originally safe, but currently punishment-associated pattern and prolonged the periods in the originally punished, but currently safe quadrant (Fig. $5 C$ ). In contrast, the flight behavior of flies with dysfunctional APL-MB circuit was distinctly different. During the reversal training session, these flies made repeated at- 

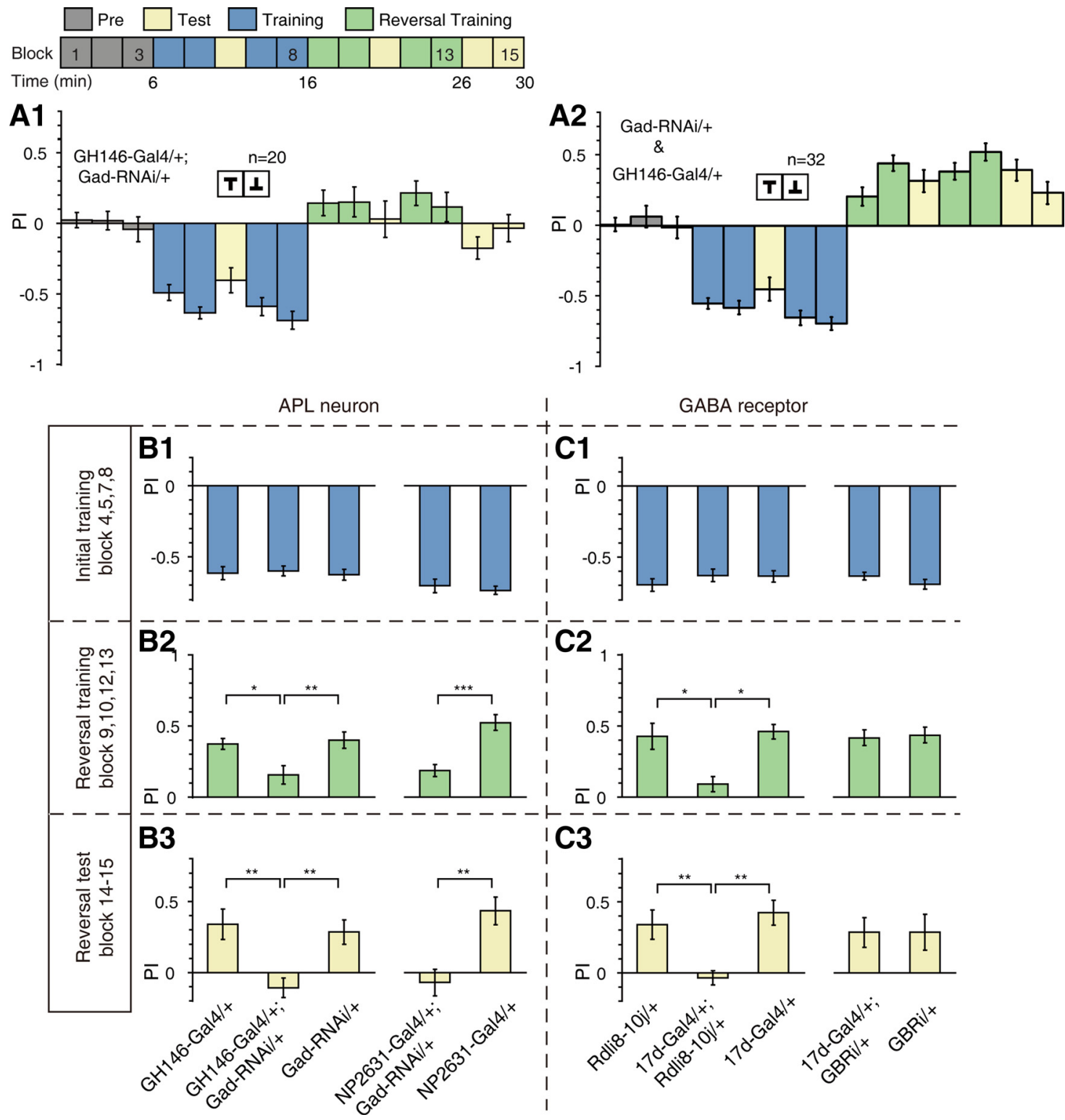

Figure 3. The APL neurons are required for visual reversal learning. A, PIs of GH146-Gal4/+;Gad-RNAi/+ (A1) and GH146-Gal4/+ and Gad-RNAi/+ (A2) flies in visual reversal learning. No difference was found between the two genetic control lines, so their PIs were pooled together. B. Summary of the effects of reduced GABA synthesis in GH146-Gal4 (left three columns) and NP2631-Gal4 (right two columns)-expressing neurons on reversal learning. $n=16-20$ flies per group. $\boldsymbol{B} 1$, No significant difference was found between Pls of the initial training session. $\boldsymbol{B 2}$, The

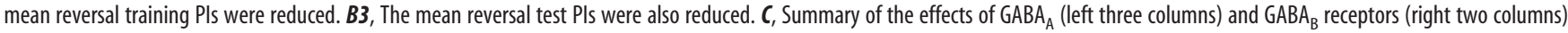
knockdown on reversal learning. $n=14-22$ flies per group. GBRi: GABA $A_{B}$ receptor R2 subunit RNAi. C1, No significant difference was found between PIs of the initial training session. C2, RDL knockdown reduced the mean reversal training $\mathrm{PI}$, whereas $\mathrm{GABA}_{B}-\mathrm{R} 2$ knockdown did not. $\mathrm{C}$, $\mathrm{RDL}$ knockdown reduced the mean reversal test $\mathrm{PI}$, whereas $\mathrm{GABA}_{B}-\mathrm{R}_{2}$ knockdown did not. Data were shown as means \pm SEM. ${ }^{*} p<0.05 ;{ }^{* *} p<0.01 ;{ }^{* * *} p<0.001$. $n$, the total number of flies examined.

tempts to stay in the newly punished quadrants despite the negative feedback, and failed to show a lasting preference for the newly safe pattern (Fig. 5D-F; data not shown). Thus, comparing with control flies, flies with dysfunctional APL-MB circuit received more punishment in reversal training. During the reversal test session, they did not exhibit an obvious preference between these two patterns (Fig. 5D-F).

\section{The APL-MB circuit is dispensable for simple forms of visual learning}

There are several alternative possibilities that may explain the impaired behavioral flexibility in flies with dysfunctional APL-MB circuit. First, flies bearing the GH146-Gal4 and GadRNAi transgenes, or flies with RDL-RNAi expression in the MB neurons, exhibit enhanced olfactory learning performance (Liu and Davis, 2009). These findings raise the possibility that the inflexibility may result from enhanced initial visual learning, which takes more training to reverse. Although the memory PI of flies with dysfunctional APL-MB circuit did not increase, this may be due to a ceiling effect of the initial training. To address this issue, we diminished the training intensity (Fig. 6A). We found that when the intensity was set to $66.7 \%$ of previous experiments, flies showed decreased training and test PI comparable to that of reversal learning (Fig. 6B). We found no significant difference between the groups with or without functional APL neurons with regard to learning or memory PIs (ANOVA, both $p>0.05$ ) (Fig. $6 B$ ). Essentially the same results were found when the punishment intensity was further reduced to the extent that wild-type 


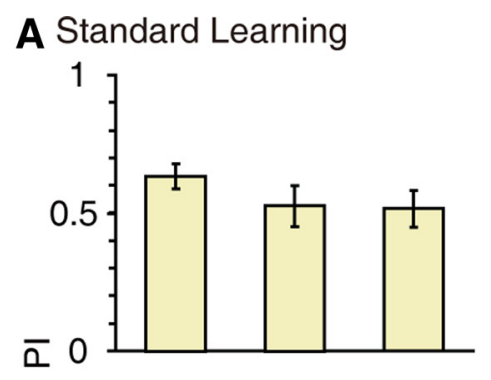

Test

\section{block 9-10}
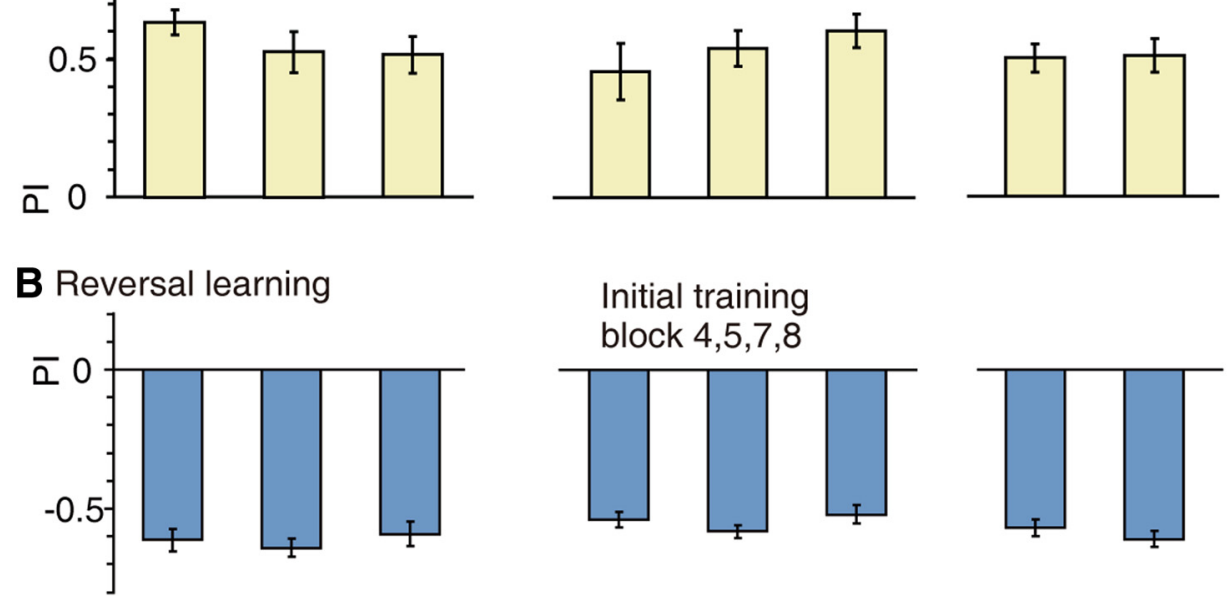

Initial training

block 4,5,7,8

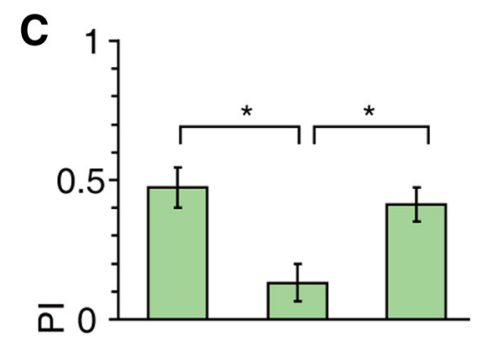

\section{Reversal training block 9,10,12,13}
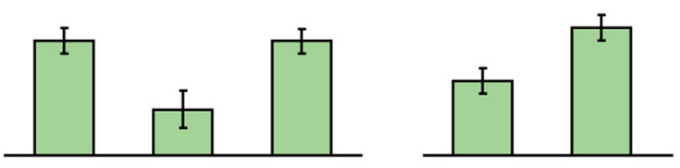

D

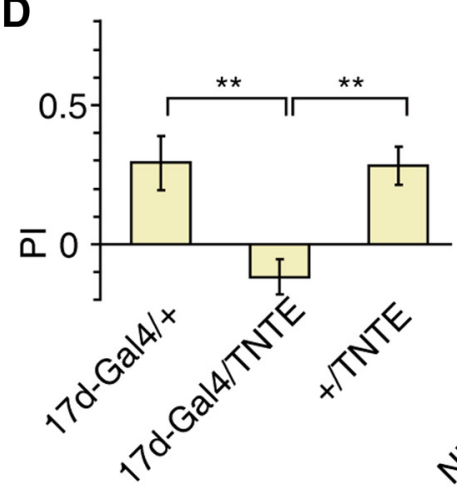

E

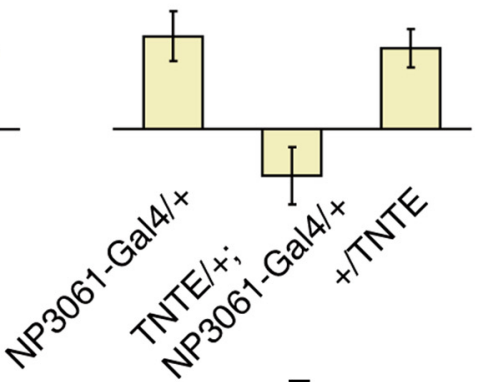

Reversal test block 14-15
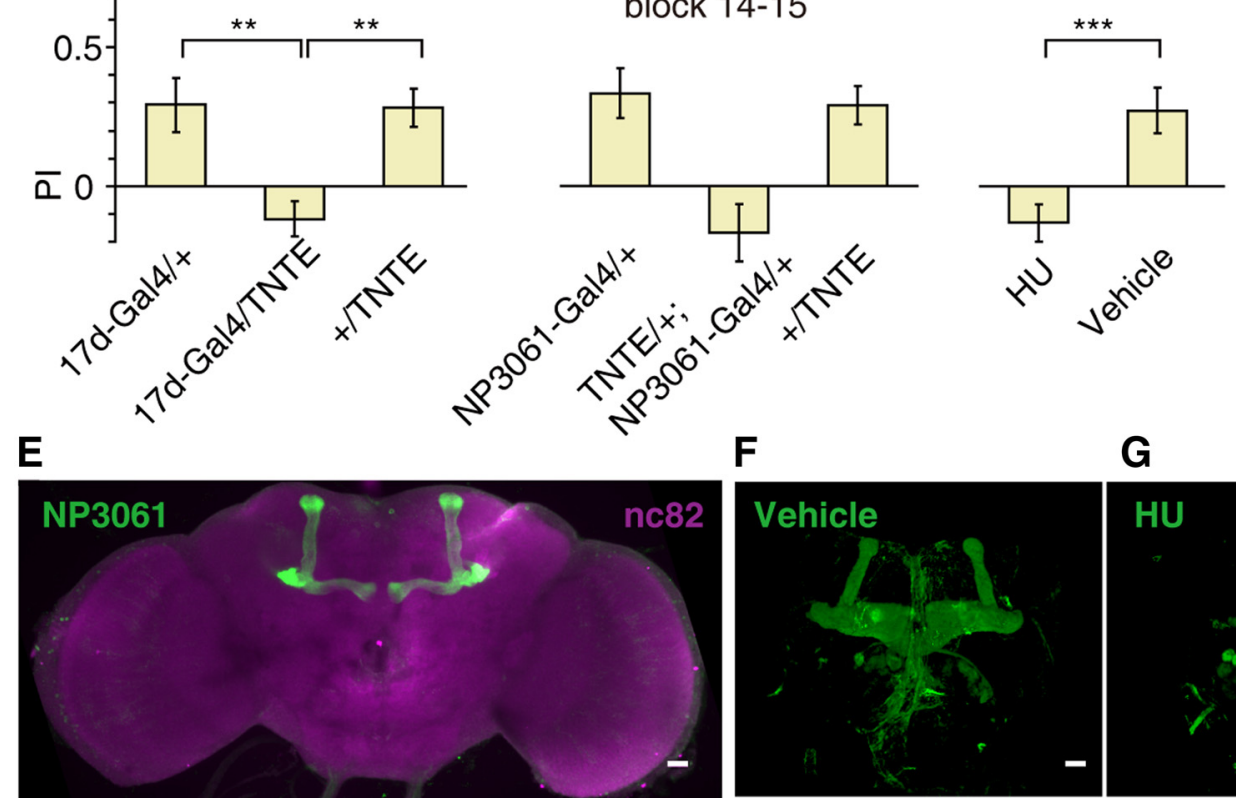

Figure 4. Specific requirement of the MB for visual reversal learning. Three methods were used to manipulate the normal function of the MBs: expression of TeTxLC in 17d-Gal4, expression of TeTxLC in NP3061-Gal4, and hydroxyurea ablation of the MBs. $A$, Manipulations of the MBs did not impair visual short-term memory. $n=12-26$ flies per group. $\boldsymbol{B}-\boldsymbol{D}$, Manipulations of the MBs have no effect on initial learning $(\boldsymbol{B})$, but did on impaired reversal training $(\boldsymbol{C})$ and reversal test $(\boldsymbol{D}) . n=14-26$ flies per group. $\boldsymbol{E}$, Adult expression pattern of the NP3061-Gal4, visualized by mCD8-GFP expression (green). Neuropils were counterstained with anti-nc82 antibody (magenta). $\boldsymbol{F}, \boldsymbol{G}$, Frontal views of two representative 3D confocal reconstructions of the fly's brain by anti-Fasll staining. $\boldsymbol{F}$, The structure of MBs was normal in vehicle group. $\boldsymbol{G}$, The majority of MBs was absent in HU group. Data were shown as means \pm SEM. ${ }^{*} p<0.05 ;{ }^{* *} p<0.01 ;{ }^{* * *} p<0.001$. Scale bars, $20 \mu \mathrm{m}$.

flies barely learn (ANOVA, both $p>0.05$ ) (Fig. 6C, 33.3\% training intensity of previous experiments). Moreover, under 66.7\% training intensity, reducing the expression of RDL receptors using the $17 \mathrm{~d}-$ Gal4 had no significant effect on the learning and memory PI (ANOVA, both $p>0.05$ ) (Fig. 6D). Chemical ablation of the MBs also left this low-intensity learning intact (twosample $t$ test, both $p>0.05$ ) (Fig. $6 E$ ). These results do not support improved learning capability as a potential explanation 

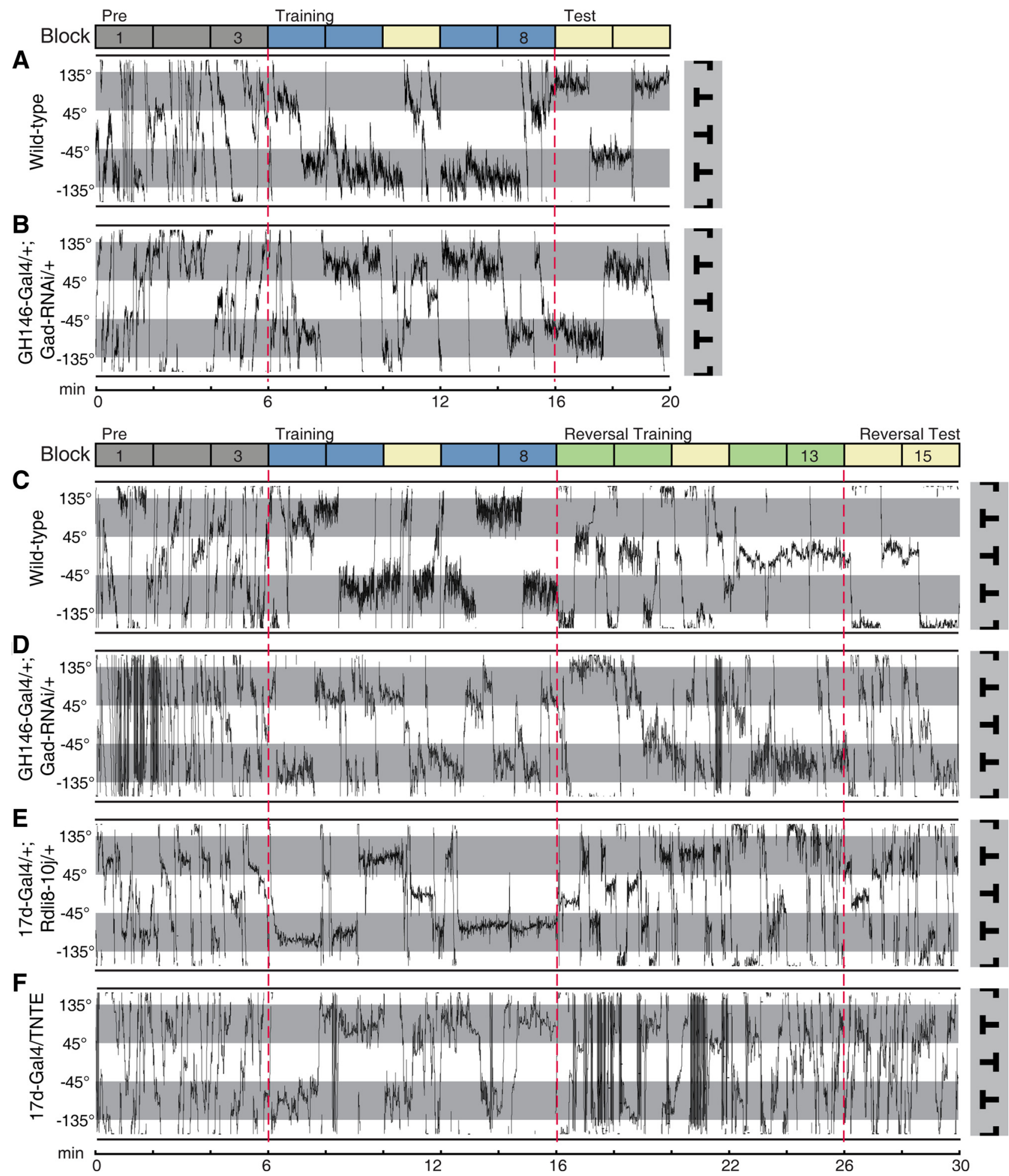

Figure 5. Flight paths during visual learning and reversal learning. $A$, A representative flight trace of individual wild-type flies during standard visual conditioning. Flight orientations in the light sectors were associated with heat during the training period (blue blocks), while flight orientations in the shading sectors represent safe flight directions. The transitions between sessions are indicated by dashed red lines. The fly was highly active, frequently shifting its orientations from one sector to another during the "pre" period (gray blocks). The flight directions were mainly restricted to the safe sector during the "training" period (blue blocks). The preference for the safe sector was maintained during the "test" period (yellow blocks), when heat was switched off. $\boldsymbol{B}$, Similar to $A$, except that the genotype is GH146-Ga/4/+; Gad-RNAi/+. C-E, Representative flight traces of individual flies during visual reversal learning. The reinforcement contingency was reversed when "reversal training" (green blocks) began. C, Shows the flight path of a wild-type fly, whose preference switched from inverted $T$ to upright $T$ after the contingency switch. $\mathbf{D}-\boldsymbol{F}$, Show the performance of three representative flies with dysfunctional APL-MB circuit. The genotype for the specific trace is shown on the left. These flies could neither effectively avoid the newly punished pattern nor maintain a preference toward the safe pattern. The flight directions were distributed almost equally in the four quadrants during the "reversal test." 


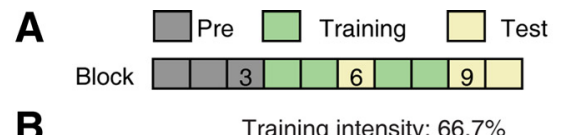

B

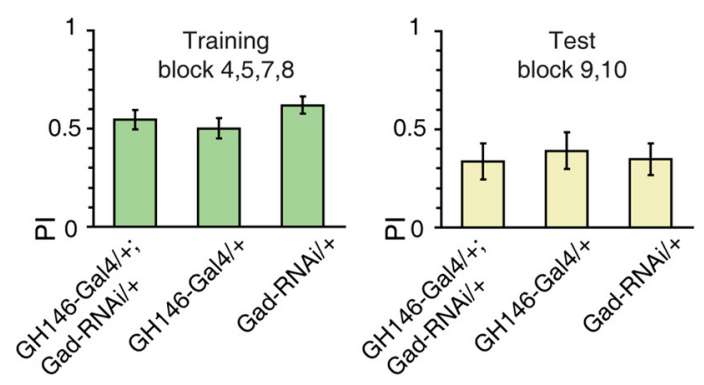

D

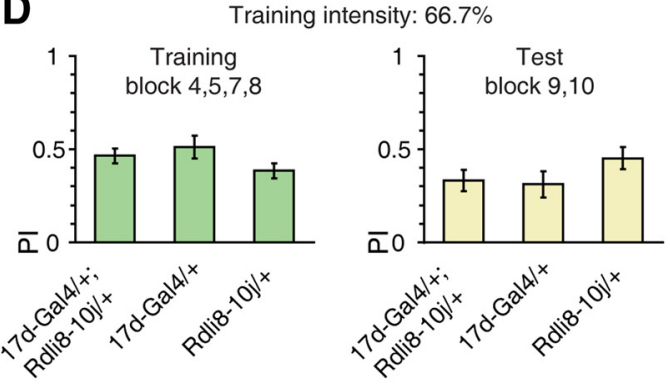

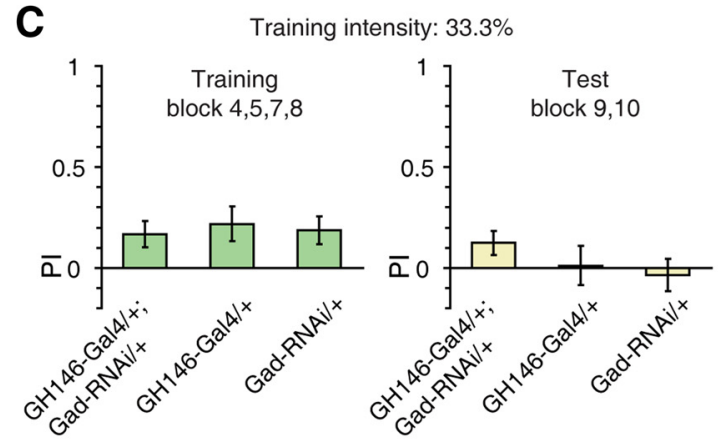

E

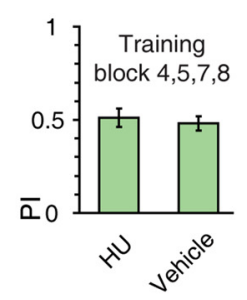

Training intensity: $66.7 \%$

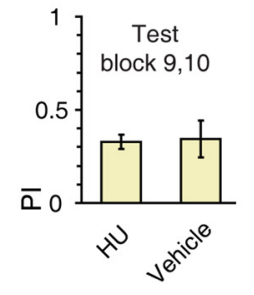

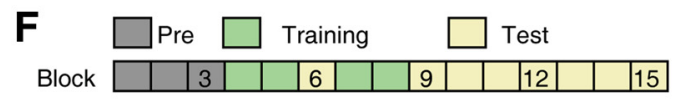

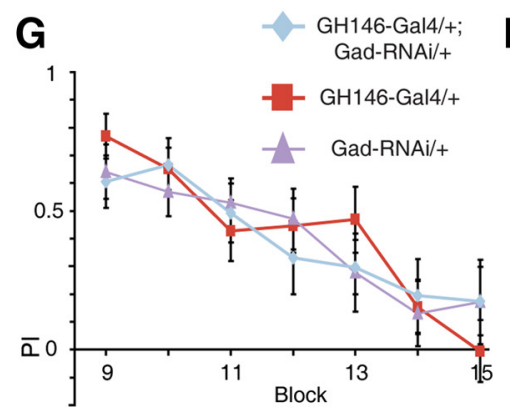

$\mathbf{H}$
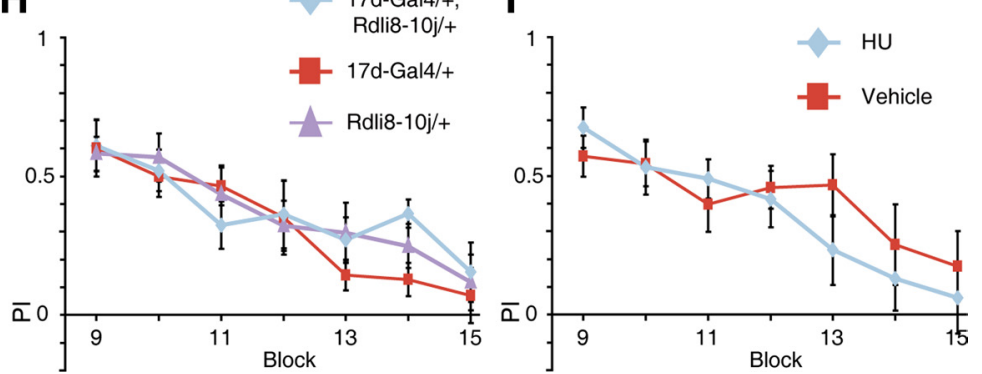

Figure 6. The APL-MB circuit is dispensable for simple visual learning and extinction. $\boldsymbol{A}$, Learning under low punishment intensity. B, Under 66.7\% training intensity, GH146-Gal4/+; Gad-RNAi/+ flies showed intact learning and short-term memory performance, comparing with GH146-Gal4/+ and Gad-RNAi/+ flies. $n=18$ flies per group. C, When the punishment intensity was further reduced to the extent that wild-type flies barely learn (33.3\% of previous experiments), the learning and test PIs did not significantly increase in GH146-Gal4/+;Gad-RNAi/+ flies. We note there was a trend for higher memory scores in the experimental flies. $n=18-20$ flies per group. $\boldsymbol{D}$, Under $66.7 \%$ training intensity, $17 \mathrm{~d}-$-Gal $4 /+;$ UAS-Rdli8 $-10 \mathrm{j} /+$ flies showed intact learning and short-term memory performance, comparing with genetic controls. $n=10$ flies per group. $E$, Under $66.7 \%$ training intensity, MB-ablated flies showed intact learning and short-term memory performance, comparing with vehicle flies. $n=14$ flies per group. $F$, Extinction assay. The final test period lasted for 14 min. $G$, The mean Pls of GH146-Gal4/+; Gad-RNAi/ + flies were not statistically different from control flies across the testing phase. $n=16$ flies per group. $\boldsymbol{H}$, The mean PIs of $17 \mathrm{~d}-G a / 4 /+;$ UAS-Rdli8 $-10 \mathrm{j} /+$ flies were not statistically different from control flies across the testing phase. $n=12$ flies per group. $I$, The mean PIs of HU flies were not statistically different from vehicle flies across the testing phase. $n=14$ flies per group. Data were shown as means \pm SEM. ${ }^{*} p<0.05 ;{ }^{* *} p<0.01 ; * * * p<0.001$.

for the inflexibility phenotype observed in previous reversal learning experiments.

Second, the inflexibility may be caused by decreased extinction of the initial memory (Devaud et al., 2007; Schiller and Delgado, 2010). To examine this possibility, we prolonged the testing phase of the simple learning task to measure the profile of memory extinction after initial training (Fig. $6 F$ ). We examined the performance of GH146-Gal4/+; Gad-RNAi/+ flies, 17dGal4/+; UAS-Rdli8-10j/+ flies, and HU flies along with relevant control flies in this task. The test PIs were examined by repeatedmeasures ANOVA with genotype as the between-subjects factor, and block number $(9-15)$ as the within-subjects factor. We found that the test PIs of all groups decreased along the $14 \mathrm{~min}$ testing period (significant main effect of block number: all $p<$ 0.001) (Fig. 6G-I). However, GH146-Gal4/+; Gad-RNAi/+ flies showed similar performance levels across the testing phase compared with genetic controls $\left(F_{(2,45)}=0.25, p=0.78\right)$ (Fig. $\left.6 G\right)$. Likewise, the performance of $17 \mathrm{~d}-$ Gal4/+; UAS-Rdli8-10j/+ flies exhibited a similar rate of reduction as that of genetic controls $\left(F_{(2,33)}=0.37, p=0.69\right)$ (Fig. $\left.6 H\right)$. In addition, the memory PI of MB-ablated flies was statistically insignificant from vehicle flies in this extinction task $\left(F_{(1,26)}=0.43, p=0.52\right)$ (Fig. $\left.6 I\right)$. There was no significant interaction between blocking number and genotype in all cases $(p>0.05)$. These results indicate that the extinction factor is unlikely to account for the poor reversal performance after dysfunction of the APL-MB circuit. 
A
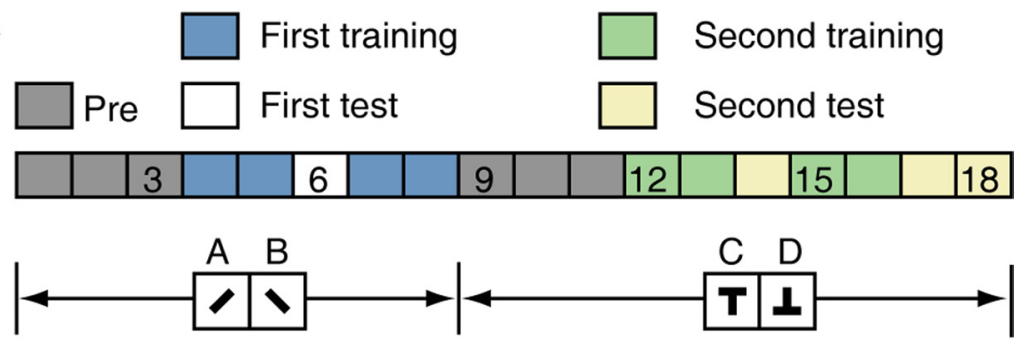

B
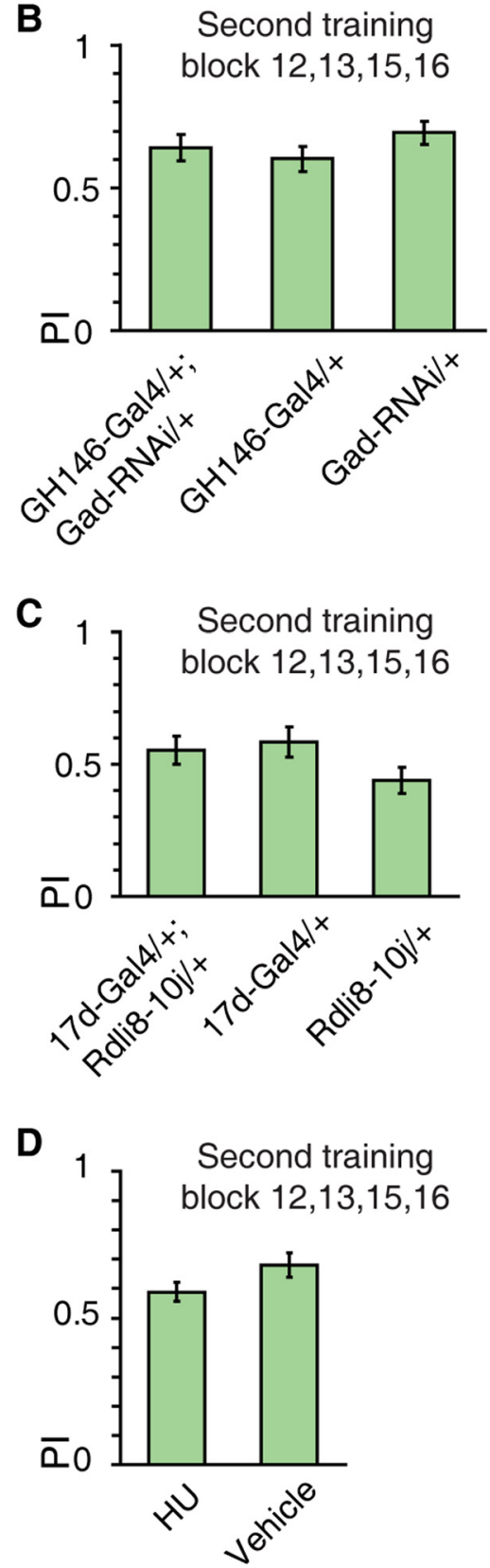
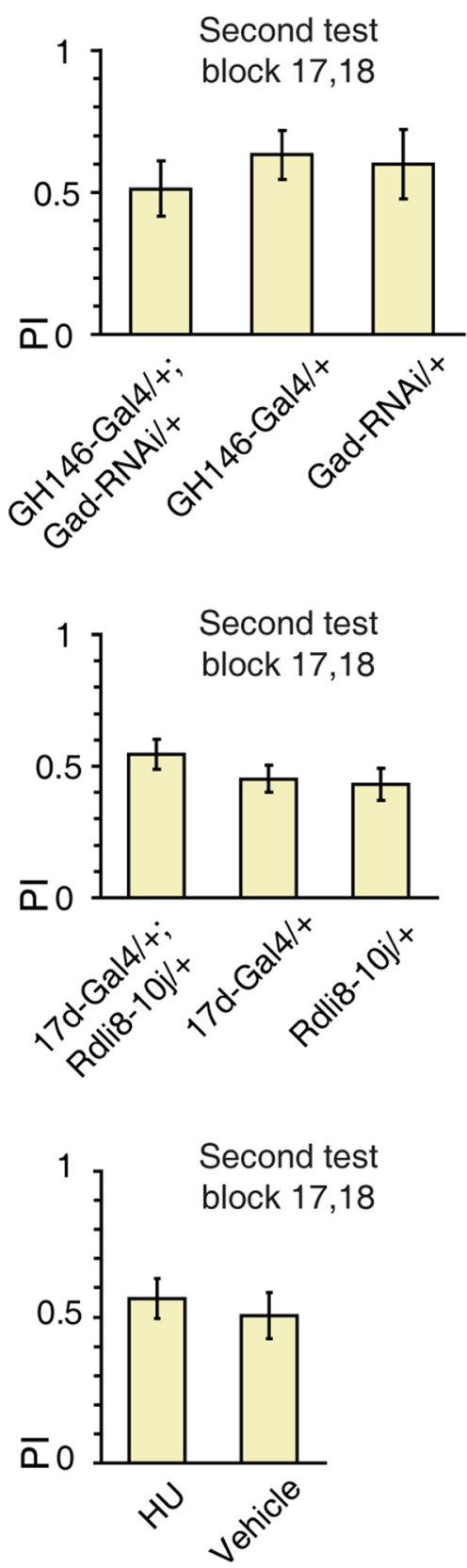

Figure 7. The APL $-M B$ circuit is dispensable for differential conditioning. $A$, Differential conditioning: the $A+, B / C+, D$ task with + indicates punishment, letters indicate visual patterns. $\boldsymbol{B}$, No significant difference had been found for the mean PIs of GH146-Gal4/+; Gad-RNAi/ + flies and control flies during the second training and test sessions. $n=8-10$ flies per group. C, No significant difference had been found for the mean PIs of $17 \mathrm{~d}-\mathrm{Ga} / 4 /+$; UAS-Rdli8 - 10j/ + flies and control flies during the second training and test sessions. $n=10$ flies per group. $D$, No significant difference had been found for the mean PIs of HU flies and vehicle flies during the second training and test sessions. $n=20-24$ flies per group. Data were shown as means \pm SEM. ${ }^{*} p<$ $0.05 ;{ }^{* *} p<0.01 ;{ }^{* * *} p<0.001$.

Third, the reversal learning deficit could stem from the possibility that flies simply could not learn a novel discrimination after first experiencing one learning session, regardless of the relationship between the two training sessions. To probe this possibility, we used a "differential conditioning" task involving novel visual stimuli in the second training phase (Fig. $7 A$; the $\mathrm{A}+, \mathrm{B} / \mathrm{C}+, \mathrm{D}$ task, with + indicate punishment, letters indicate visual patterns) (Devaud et al., 2007). We sequentially trained flies using distinct visual parameters (contour orientation and vertical position of center of gravity) in two sessions. A 6 min testing period was added before the second training session to familiarize the flies with the new patterns (Fig. 7A). Control experiments showed that, after addition of this testing period, the reversal training and test PI of CS flies ( $0.34 \pm 0.05$ and $0.28 \pm 0.07$, respectively) was not significantly different from that of standard reversal learning shown in Figure $1 C$ (two-sample $t$ test, both $p>0.05$ ). We found that the performance of GH146-Gal4/+; Gad-RNAi/+ flies was statistically indistinguishable from control flies in the second training and test sessions (ANOVA, both $p>0.05$ ) (Fig. $7 B$ ). Along this line, expression of UAS-Rdli8-10j in 17d-Gal4 did not affect the performance of the second training and test sessions (ANOVA, both $p>0.05$ ) (Fig. 7C). Chemical ablation of the MBs also had little impact on the ability of flies to learn a second discrimination (twosample $t$ test, both $p>0.05$ ) (Fig. 7D). These results indicate that the reversal deficit is unlikely due to a general failure in learning a novel discrimination after one previous training session.

Together, the above results support the notion that APL-MB circuit is potentially dispensable for simple forms of visual learning such as initial learning, extinction, and differential conditioning.

Acute requirement of the APL-MB circuit for visual reversal learning To determine whether the decrement in reversal performance was the consequence of acute disruptions or developmental insults, we used the TARGET system (McGuire et al., 2003), in which the temporal expression of the Gal4-UAS system is controlled by a temperaturesensitive Gal80 ${ }^{\text {ts }}$ under the control of a ubiquitously expressing tubulin promoter (tub-Gal $80^{\text {ts }}$ ). The Gal $80{ }^{\text {ts }}$ protein is functional and represses the transcriptional activity of Gal4 at the PT $\left(18^{\circ} \mathrm{C}\right)$, whereas it becomes dysfunctional and re- 

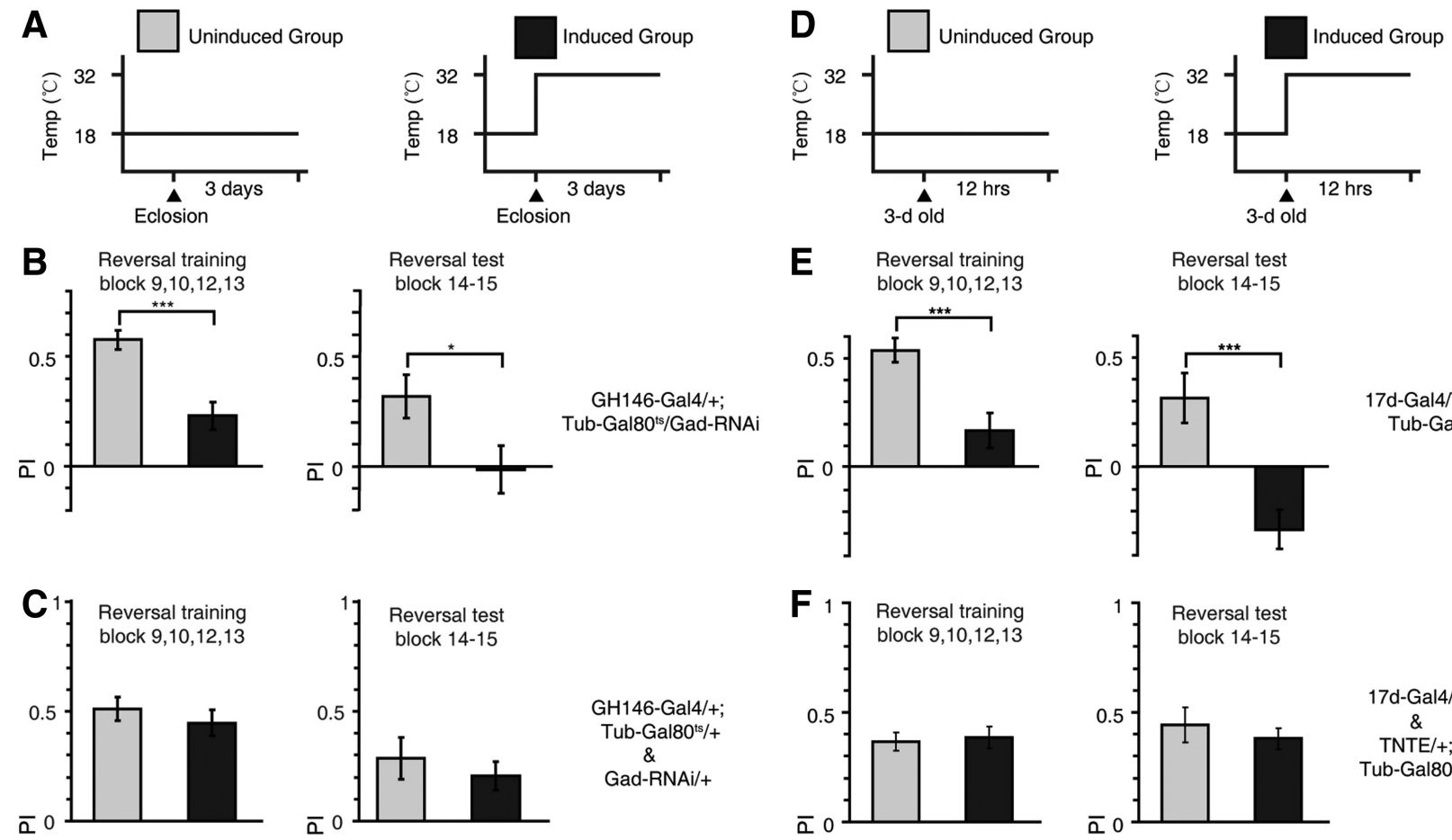

Reversal test block 14-15
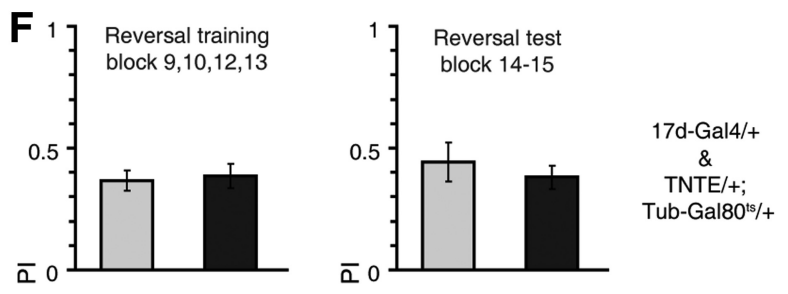

Figure 8. The APL-MB circuit is required post developmentally for reversal learning. $A$, Heat shock regime: the uninduced group stayed at PT throughout development and adulthood (PT $18^{\circ} \mathrm{C}$ ). The induced group was transferred to $\mathrm{RT}\left(30^{\circ} \mathrm{C}\right)$ for $3 \mathrm{~d}$ to induce transgene expression after the development phase in PT. The induced group was tested in the reversal learning task within $0-6 \mathrm{~h}$ after the heat shock treatment. $\boldsymbol{B}$, The mean reversal training and test PIs were reduced after Gad-RNAi induction in GH146-Gal4. $n=14-20$ flies per group. $\boldsymbol{C}$, After heat shock, the mean reversal training and test Pls were statistically indistinguishable from uninduced controls for both GH146-Gal4/+;Tub-Gal80 ${ }^{\text {s }} /+$ and Gad-RNAi/ + flies. No significant difference had been found between these two genotypes, so their data were pooled together. $n=20$ flies per group. $\boldsymbol{D}$, Same as $\boldsymbol{A}$, except that the heat shock lasted for $12 \mathrm{~h}$ and flies were transferred into $R T$ when $3 \mathrm{~d}$ old. $\boldsymbol{E}$, The mean reversal training and test PI were reduced after TNTE induction in $17 \mathrm{~d}-G a l 4 . n=14$ flies per group. Also note that, for unknown reasons, the reversal test Pl is negative. $F$, After heat shock, the mean reversal training and test PI were statistically indistinguishable from uninduced controls for both $17-G a / 4 /+$ and TNTE/ +; Tub-Gal 80 ts $/+$ flies. No significant difference had been found between these two genotypes, so their data were pooled together. $n=20$ flies per group. Data were shown as means \pm SEM. ${ }^{*} p<0.05 ;{ }^{* *} p<0.01 ;{ }^{* * *} p<0.001$.

leases the Gal4-UAS system from repression above the $\mathrm{RT}\left(30^{\circ} \mathrm{C}\right)$. For each genotype, flies were divided into two treatment groups. One control group was kept at the PT throughout development and adulthood, whereas a second group of the same genotype was raised at the $\mathrm{PT}$ until eclosion and transferred to the RT to induce UAS-transgene expression (Fig. $8 A$ ).

The reversal training and test performance were impaired in flies expressing Gad-RNAi in the GH146-Gal4 for $3 \mathrm{~d}$ in the adult phase, compared with that of genetically identical flies without such Gad-RNAi expression (two-sample $t$ test, $t_{(32)}=4.50, p<$ 0.001 and $t_{(32)}=2.24, p=0.03$, respectively) (Fig. $8 B$ ). To exclude the possibility that heat shock alone had any effect, we tested the genetic control lines $\left(\mathrm{GH} 146 /+\right.$; Tub-Gal $80^{\text {ts }} /+$ and Gad-RNAi/+) in the reversal task after heat shock treatment and found that they showed wild-type reversal performance (twosample $t$ test, both $p>0.05$ ) (Fig. 8C). Acute expression of TNTE in the $17 \mathrm{~d}-$ Gal4 by heat shock treatment for $12 \mathrm{~h}$ in the adult phase (Fig. $8 D$ ) also disrupted normal reversal learning performance (two-sample $t$ test, $t_{(26)}=3.78, p<0.001$ and $t_{(26)}=4.15$, $p<0.001$, respectively) (Fig. $8 E$ ). The same heat shock treatment did not impair reversal learning in genetic control strains $(17 \mathrm{~d}$ Gal4/+ and TNTE/+; tub-Gal80 ${ }^{\text {ts }} /+$ ) (two-sample $t$ test, both $p>0.05$ ) (Fig. $8 F$ ). Together, the above results indicate that the APL-MB circuit is required acutely for reversal learning.

\section{Discussion}

Using the flight simulator, here we have established a visual reversal learning paradigm to assess the profile of flexible behavior in Drosophila and demonstrated that flies possess remarkable be- havioral flexibility when facing environmental changes. We found that knocking down GABA synthesis in the APL neurons, which has broad areas of innervations throughout the MBs, produced an impairment in visual reversal learning, suggesting that GABA release from the APL neurons onto the MBs promotes behavioral flexibility. This interpretation was supported by the observation that RNA interference-mediated downregulation of ionotropic $\mathrm{GABA}_{\mathrm{A}}$ receptors in the $\mathrm{MB} \alpha / \beta$ lobe caused a specific reversal learning deficit. Perturbation of the output of the MBs by TNT expression or chemical ablation of the MBs also abolished reversal learning. Thus, these three lines of evidence support the hypothesis that the APL neurons and the MBs constitute an inhibitory neural circuit that is necessary for flexible control of behavior in a volatile environment. In addition, the TARGET system enabled us to show that the APL-MB circuit is required post developmentally for reversal learning.

In our experiments, we found that flies with defective APL-MB circuit exhibited no obvious preference among the visual patterns during the reversal test (except for Fig. $8 E$ ). There are several possible mechanisms underlying this behavioral effect. One possibility is that these flies failed to learn the reversed association, while forgetting or erasing their initial memory during reversal training. Consistent with this notion, a previous study reported that both reversal learning and forgetting require Rac signaling in the MBs, suggesting that these two processes might have certain overlapping components (Shuai et al., 2010). Forgetting had traditionally been viewed as a failure to preserve useful information, and thus undesir- 
able. Yet it has been proposed that forgetting might be adaptive for animals living in a changing environment (Kraemer and Golding, 1997). This proposition is exemplified in our reversal learning task, during which forgetting was advantageous when actions based on the initial memory were harmful after the contingency switch.

There also exists the possibility that the reversal test behavior of flies with defective APL-MB circuit stem from a failure to decide between the new association and the old one. Although these flies exhibited a reduced reversal training PI, they might have, to some extent, acquired the new association after receiving more reinforcement than genetic controls. And it is likely that the initial memory was only temporarily suppressed by reversal training instead of being permanently deleted, and was released from suppression during reversal test. In this case, wild-type flies might be capable of using additional cues, such as the temporal relationship of the respective memory, to correctly decide which association to follow, whereas flies with defective APL-MB circuit are indecisive among the conflicting memories. In line with this speculation, our previous study showed that flies with defective dopamine-MB circuit have trouble deciding between two conflicting visual features (Tang and Guo, 2001; Zhang et al., 2007). Future systematic investigations are required to reveal the exact neural underpinning of the inflexible behavior of flies with defective APL-MB circuit.

In Drosophila, the MBs have been thought to be mainly involved in olfactory learning, and were hypothesized to be the site for olfactory memory storage (Keene and Waddell, 2007; Heisenberg and Gerber, 2008; Davis, 2011). Compared with olfaction, the role of MBs in processing information from other modalities has been much less extensively studied. Although dispensable for basic visual learning (Wolf et al., 1998), accumulating evidence points to the critical involvement of the MBs in a variety of visual "cognitive" behaviors, such as contextual generalization (Liu et al., 1999), visual selective attention (van Swinderen and Greenspan, 2003; Xi et al., 2008; van Swinderen et al., 2009), feature extraction (Peng et al., 2007), and multimodal sensorimotor transformation (Chow et al., 2011). Interestingly, recent data indicate that the MBs mediate the inhibition of the operant learning system by the classical learning system (Brembs, 2009). Such an inhibitory effect could prevent premature habit formation and promote generalization of predictive information during composite learning situations (Brembs, 2009). It is worth noting that the task for habit formation also involves changes in task contingency (from stimulus-reinforcement to action-reinforcement). Thus, the contribution of the MBs to habit formation and reversal learning may potentially be very similar. These findings imply the possibilities that the underlying circuits for habit formation and reversal learning might be shared in Drosophila and future research may test whether the APL neurons also play an important role in habit formation.

A previous study revealed that honeybees with silenced MBs failed an olfactory reversal learning task but still retained the capability of performing extinction and differential conditioning tasks (Devaud et al., 2007). Based on these findings, Devaud et al. (2007) proposed that MBs might be specifically required for the resolution of conflicting information, which is only present in the reversal task. Therefore, our findings extended these results into the visual system of the fruit flies, suggesting that MBs might have a general function of resolving conflicting information. At present, we know little about how conflict resolution could be achieved mechanistically in the MBs. Based on the results of our previous studies, we hypothesized that a gating mechanism is implemented in the MBs that inhibits the weaker inputs and amplifies the stronger inputs (Peng et al., 2007; Zhang et al., 2007; Xi et al., 2008). In this study, we have identified the APL neurons as a potential candidate that might mediate this gating process. In locust, the giant GABAergic neurons, a pair of neurons structurally analogous to the APL neurons, was found to form an all-toall negative-feedback loop with the ipsilateral $\mathrm{MB}$ neurons (Papadopoulou et al., 2011). If the APL neurons also form a feedback loop with the MBs, they could potentially filter the incoming inputs, and select the most appropriate ones through intrastimulus competition based on saliency, motivational value, or temporal factors. In addition, the APL neurons form heterotypic gap junctions with the dorsal paired medial neurons (Wu et al., 2011) and may also affect learning through its interactions with the diverse neuromodulator systems projecting to the MBs (Tanaka et al., 2008; Mao and Davis, 2009; Chiang et al., 2010). Hopefully, future investigation combining behavioral paradigms with electrophysiological (Maimon et al., 2010) or imaging techniques (Seelig et al., 2010) would shed more light onto this intriguing issue.

In this study, we used two approaches to perturb the normal function of the APL-MB circuit: blocking the inhibitory input of the MBs, which presumably would lead to increased output of the MBs, as well as silencing the output of MBs. These two opposite manipulations of the MB output nevertheless both produced a reversal learning deficit. This discrepancy might be reconciled by the possibility that these two manipulations both lead to a disruption of the optimal level of MB output. Excitation-inhibition balance is known to be critical in the operation of a wide variety of neural circuits (Rubenstein and Merzenich, 2003; Haider et al., 2006), and an optimal level of balance between excitatory and inhibitory inputs may be critical for the operation of $\mathrm{MB}$ circuit (Papadopoulou et al., 2011). Blocking the inhibitory inputs to $\mathrm{MB}$ might disrupt this balance and result in a dysfunctional $\mathrm{MB}$ output that is unable to support reversal learning. In mammalian systems, reducing the number of GABAergic interneurons in the OFC also disrupted reversal learning (Bissonette et al., 2010), similar to the phenotype produced by OFC lesion (Schoenbaum et al., 2003). Together, these findings support the notion that an optimal level of circuit output is crucial for neural operations underlying flexible behavior.

In the present study, we found that dysfunction of an inhibitory circuit impaired the ability of flies to rapidly adapt behavior to changing environmental contingencies. Previous studies of the role of APL neurons in olfactory learning found that when GABA synthesis was reduced in the APL neurons, the learning score was enhanced (Liu and Davis, 2009). This result was surprising because it seems that enhanced learning abilities would bring selective advantages to the organism and should be used in nature. Our findings support the idea that the role of an inhibitory system in learning might be to ensure that an optimal level of memory strength is formed, such that it could be rapidly reversed when the environmental contingency is changed. We believe that our visual reversal learning paradigm can be used as a simple model for assessing flexible behavior in Drosophila. We hope that the power of Drosophila genetics, together with emerging new techniques for monitoring and manipulating neural activity (Venken et al., 2011), will allow us to gain more insight into behavioral flexibility at the molecular and circuit level.

\section{References}

Aso Y, Grübel K, Busch S, Friedrich AB, Siwanowicz I, Tanimoto H (2009) The mushroom body of adult Drosophila characterized by GAL4 drivers. J Neurogenet 23:156-172. 
Bissonette GB, Bae MH, Suresh T, Jaffe DE, Powell EM (2010) Astrocytemediated hepatocyte growth factor/scatter factor supplementation restores GABAergic interneurons and corrects reversal learning deficits in mice. J Neurosci 30:2918-2923.

Brembs B (2009) Mushroom bodies regulate habit formation in Drosophila. Curr Biol 19:1351-1355.

Brembs B, Wiener J (2006) Context and occasion setting in Drosophila visual learning. Learn Mem 13:618-628.

Brigman JL, Graybeal C, Holmes A (2010) Predictably irrational: assaying cognitive inflexibility in mouse models of schizophrenia. Front Neurosci $4: 13$

Chamberlain SR, Menzies L, Hampshire A, Suckling J, Fineberg NA, del Campo N, Aitken M, Craig K, Owen AM, Bullmore ET, Robbins TW, Sahakian BJ (2008) Orbitofrontal dysfunction in patients with obsessive-compulsive disorder and their unaffected relatives. Science 321:421-422.

Chiang AS, Lin CY, Chuang CC, Chang HM, Hsieh CH, Yeh CW, Shih CT, Wu JJ, et al. (2011) Three-dimensional reconstruction of brain-wide wiring networks in Drosophila at single-cell resolution. Curr Biol 21:1-11.

Chow DM, Theobald JC, Frye MA (2011) An olfactory circuit increases the fidelity of visual behavior. J Neurosci 31:15035-15047.

Clarke HF, Roberts AC (2011) Reversal learning in fronto-striatal circuits: a functional, autonomic, and neurochemical analysis. In: Decision making, affect, and learning (Delgado M, Phelps EA, Robbins TW eds.), pp 205235. New York: Oxford UP.

Davis RL (2005) Olfactory memory formation in Drosophila: from molecular to systems neuroscience. Ann Rev Neurosci 28:275-302.

Davis RL (2011) Traces of Drosophila memory. Neuron 70:8-19.

de Belle IS, Heisenberg M (1994) Associative odor learning in Drosophila abolished by chemical ablation of mushroom bodies. Science 263:692-695.

Devaud JM, Blunk A, Podufall J, Giurfa M, Grünewald B (2007) Using local anaesthetics to block neuronal activity and map specific learning tasks to the mushroom bodies of an insect brain. Eur J Neurosci 26:3193-3206.

Dias R, Robbins TW, Roberts AC (1996) Dissociation in prefrontal cortex of affective and attentional shifts. Nature 380:69-72.

Dietzl G, Chen D, Schnorrer F, Su KC, Barinova Y, Fellner M, Gasser B, Kinsey K, Oppel S, Scheiblauer S, Couto A, Marra V, Keleman K, Dickson BJ (2007) A genome-wide transgenic RNAi library for conditional gene inactivation in Drosophila. Nature 448:151-156.

Enell L, Hamasaka Y, Kolodziejczyk A, Nässel DR (2007) Gammaaminobutyric acid (GABA) signaling components in Drosophila: immunocytochemical localization of $\mathrm{GABA}_{\mathrm{B}}$ receptors in relation to the $\mathrm{GABA}_{\mathrm{A}}$ receptor subunit RDL and a vesicular GABA transporter. J Comp Neurol 505:18-31.

Fellows LK, Farah MJ (2003) Ventromedial frontal cortex mediates affective shifting in humans: evidence from a reversal learning paradigm. Brain 126:1830-1837.

Götz KG (1964) Optomotorische untersuchung des visuellen systems einiger augenmutanten der fruchtfliege Drosophila. Kybernetik 2:77-92.

Guo A, Li L, Xia SZ, Feng CH, Wolf R, Heisenberg M (1996) Conditioned visual flight orientation in Drosophila: dependence on age, practice, and diet. Learn Mem 3:49-59.

Haider B, Duque A, Hasenstaub AR, McCormick DA (2006) Neocortical network activity in vivo is generated through a dynamic balance of excitation and inhibition. J Neurosci 26:4535-4545.

Heimbeck G, Bugnon V, Gendre N, Keller A, Stocker RF (2001) A central neural circuit for experience-independent olfactory and courtship behavior in Drosophila melanogaster. Proc Natl Acad Sci U S A 98:15336-15341.

Heisenberg M (2003) Mushroom body memoir: from maps to models. Nat Rev Neurosci 4:266-275.

Heisenberg M, Gerber B (2008) Behavioral analysis of learning and memory in Drosophila. In: Learning and memory: a comprehensive reference (Menzel R, Byrne J, eds), pp 549-559. Oxford, UK: Elsevier.

Heisenberg M, Wolf R (1979) On the fine structure of yaw torque in visual flight orientation of Drosophila melanogaster. J Comp Physiol, A 130:113-130.

Iversen SD, Mishkin M (1970) Perseverative interference in monkeys following selective lesions of the inferior prefrontal convexity. Exp Brain Res $11: 376-386$
Keene AC, Waddell S (2007) Drosophila olfactory memory: single genes to complex neural circuits. Nat Rev Neurosci 8:341-354.

Kitamoto T (2001) Conditional modification of behavior in Drosophila by targeted expression of a temperature-sensitive shibire allele in defined neurons. J Neurobiol 47:81-92.

Komischke B, Giurfa M, Lachnit H, Malun D (2002) Successive olfactory reversal learning in honeybees. Learn Mem 9:122-129.

Kraemer PJ, Golding JM (1997) Adaptive forgetting in animals. Psychon Bull Rev 4:480-491.

Liu G, Seiler H, Wen A, Zars T, Ito K, Wolf R, Heisenberg M, Liu L (2006) Distinct memory traces for two visual features in the Drosophila brain. Nature 439:551-556.

Liu L, Wolf R, Ernst R, Heisenberg M (1999) Context generalization in Drosophila visual learning requires the mushroom bodies. Nature 400:753-756.

Liu X, Davis RL (2009) The GABAergic anterior paired lateral neuron suppresses and is suppressed by olfactory learning. Nat Neurosci 12:53-59.

Liu X, Krause WC, Davis RL (2007) $\mathrm{GABA}_{\mathrm{A}}$ receptor RDL inhibits Drosophila olfactory associative learning. Neuron 56:1090-1102.

Maimon G, Straw AD, Dickinson MH (2010) Active flight increases the gain of visual motion processing in Drosophila. Nat Neurosci 13:393-399.

Mao Z, Davis RL (2009) Eight different types of dopaminergic neurons innervate the Drosophila mushroom body neuropil: anatomical and physiological heterogeneity. Front Neural Circuits 3:5.

McGuire SE, Le PT, Osborn AJ, Matsumoto K, Davis RL (2003) Spatiotemporal rescue of memory dysfunction in Drosophila. Science 302:1765-1768.

McGuire SE, Deshazer M, Davis RL (2005) Thirty years of olfactory learning and memory research in Drosophila melanogaster. Prog Neurobiol 76:328-347

Menzel R (1969) Das Gedächtnis der Honigbiene für Spektralfarben. II. Umlernen und Mehrfachlernen. Z Vergl Physiol 63:290-309.

Papadopoulou M, Cassenaer S, Nowotny T, Laurent G (2011) Normalization for sparse encoding of odors by a wide-field interneuron. Science 332:721-725

Peng Y, Xi W, Zhang W, Zhang K, Guo A (2007) Experience improves feature extraction in Drosophila. J Neurosci 27:5139-5145.

Pfaffl MW (2001) A new mathematical model for relative quantification in real-time RT-PCR. Nucleic Acids Res 29:e45.

Pitman JL, Huetteroth W, Burke CJ, Krashes MJ, Lai SL, Lee T, Waddell S (2011) A pair of inhibitory neurons are required to sustain labile memory in the Drosophila mushroom body. Curr Biol 21:855-861.

Quinn WG, Harris WA, Benzer S (1974) Conditioned behavior in Drosophila melanogaster. Proc Natl Acad Sci U S A 71:708-712.

Reichardt W, Wenking H (1969) Optical detection and fixation of objects by fixed flying flies. Naturwissenschaften 56:424-425.

Rubenstein J, Merzenich M (2003) Model of autism: increased ratio of excitation/inhibition in key neural systems. Genes Brain Behav 2:255-267.

Schiller D, Delgado MR (2010) Overlapping neural systems mediating extinction, reversal and regulation of fear. Trends Cogn Sci 14:268-276.

Schoenbaum G, Setlow B, Nugent SL, Saddoris MP, Gallagher M (2003) Lesions of orbitofrontal cortex and basolateral amygdala complex disrupt acquisition of odor-guided discriminations and reversals. Learn Mem 10:129-140.

Schoenbaum G, Roesch MR, Stalnaker TA, Takahashi YK (2009) A new perspective on the role of the orbitofrontal cortex in adaptive behaviour. Nat Rev Neurosci 10:885-892.

Schwaerzel M, Heisenberg M, Zars T (2002) Extinction antagonizes olfactory memory at the subcellular level. Neuron 35:951-960.

Seelig JD, Chiappe ME, Lott GK, Dutta A, Osborne JE, Reiser MB, Jayaraman V (2010) Two-photon calcium imaging from head-fixed Drosophila during optomotor walking behavior. Nat Methods 7:535-540.

Shuai Y, Lu B, Hu Y, Wang L, Sun K, Zhong Y (2010) Forgetting is regulated through Rac activity in Drosophila. Cell 140:579-589.

Stalnaker TA, Takahashi Y, Roesch MR, Schoenbaum G (2009) Neural substrates of cognitive inflexibility after chronic cocaine exposure. Neuropharmacology 56 [Suppl 1]:63-72.

Stocker RF, Heimbeck G, Gendre N, de Belle JS (1997) Neuroblast ablation in Drosophila P[GAL4] lines reveals origins of olfactory interneurons. J Neurobiol 32:443-456. 
Strausfeld NJ, Hansen L, Li Y, Gomez RS, Ito K (1998) Evolution, discovery, and interpretations of arthropod mushroom bodies. Learn Mem 5:11-37.

Sweeney ST, Broadie K, Keane J, Niemann H, O'Kane CJ (1995) Targeted expression of tetanus toxin light chain in Drosophila specifically eliminates synaptic transmission and causes behavioral defects. Neuron 14:341-351.

Tanaka NK, Tanimoto H, Ito K (2008) Neuronal assemblies of the Drosophila mushroom body. J Comp Neurol 508:711-755.

Tang S, Guo A (2001) Choice behavior of Drosophila facing contradictory visual cues. Science 294:1543-1547.

Tully T, Boynton S, Brandes C, Dura JM, Mihalek R, Preat T, Villella A (1990) Genetic dissection of memory formation in Drosophila melanogaster. Cold Spring Harb Symp Quant Biol 55:203-211.

van Swinderen B, Greenspan RJ (2003) Salience modulates 20-30 Hz brain activity in Drosophila. Nat Neurosci 6:579-586.

van Swinderen B, McCartney A, Kauffman S, Flores K, Agrawal K, Wagner J, Paulk A (2009) Shared visual attention and memory systems in the Drosophila brain. PLoS One 4:e5989.
Venken KJ, Simpson JH, Bellen HJ (2011) Genetic manipulation of genes and cells in the nervous system of the fruit fly. Neuron 72:202-230.

Wolf R, Heisenberg M (1991) Basic organization of operant behavior as revealed in Drosophila flight orientation. J Comp Physiol A 169: $699-705$.

Wolf R, Wittig T, Liu L, Wustmann G, Eyding D, Heisenberg M (1998) Drosophila mushroom bodies are dispensable for visual, tactile, and motor learning. Learn Mem 5:166-178.

Wu CL, Shih MF, Lai JS, Yang HT, Turner GC, Chen L, Chiang AS (2011) Heterotypic gap junctions between two neurons in the Drosophila brain are critical for memory. Curr Biol 21:848-854.

Xi W, Peng Y, Guo J, Ye Y, Zhang K, Yu F, Guo A (2008) Mushroom bodies modulate salience-based selective fixation behavior in Drosophila. Eur J Neurosci 27:1441-1451.

Zhang K, Guo JZ, Peng Y, Xi W, Guo A (2007) Dopamine-mushroom body circuit regulates saliency-based decision-making in Drosophila. Science 316:1901-1904. 